\title{
Curating Metal-Organic Frameworks to Compose Robust Gas Sensor Arrays in Dilute Conditions
}

Arni Sturluson, Rachel Sousa, Yujing Zhang, Melanie T. Huynh, Caleb Laird, Arthur H. P. York, Carson Silsby, Chih-Hung Chang, Cory Simon

Submitted date: 26/07/2019 - Posted date: 26/07/2019

Licence: CC BY-NC-ND 4.0

Citation information: Sturluson, Arni; Sousa, Rachel; Zhang, Yujing; Huynh, Melanie T.; Laird, Caleb; York, Arthur H. P.; et al. (2019): Curating Metal-Organic Frameworks to Compose Robust Gas Sensor Arrays in Dilute Conditions. ChemRxiv. Preprint.

Metal-organic frameworks (MOFs)-- tunable, nano-porous materials-- are alluring recognition elements for gas sensing. Mimicking human olfaction, an array of cross-sensitive, MOF-based sensors could enable analyte detection in complex, variable gas mixtures containing confounding gas species. Herein, we address the question: given a set of MOF candidates and their adsorption properties, how do we select the optimal subset to compose a sensor array that accurately and robustly predicts the gas composition via monitoring the adsorbed mass in each MOF? We first mathematically formulate the MOF-based sensor array problem under dilute conditions. Instructively, the sensor array can be viewed as a linear map from gas composition space to sensor array response space defined by the matrix $\mathrm{H}$ of Henry coefficients of the gases in the MOFs. Characterizing this mapping, the singular value decomposition of $\mathrm{H}$ is a useful tool for evaluating MOF subsets for sensor arrays, as it determines the sensitivity of the predicted gas composition to measurement error, quantifies the magnitude of the response to changes in composition, and recovers which direction in gas composition space elicits the largest/smallest response. To illustrate, on the basis of experimental adsorption data, we curate MOFs for a sensor array with the objective of determining the concentration of $\mathrm{CO}_{2}$ and $\mathrm{SO}_{2}$ in the gas phase.

File list (2)

Curating_MOFs_for_sensor_arrays.pdf (20.01 MiB) view on ChemRxiv • download file Curating_MOFs_for_sensor_arrays_SI.pdf (2.14 MiB) view on ChemRxiv • download file 


\title{
Curating metal-organic frameworks to compose robust gas sensor arrays in dilute conditions
}

\author{
Arni Sturluson, Rachel Sousa, Yujing Zhang, Melanie T. Huynh, Caleb Laird, \\ Arthur H. P. York, Carson Silsby, Chih-Hung Chang, and Cory M. Simon* \\ Oregon State University \\ School of Chemical, Biological, and Environmental Engineering. Corvallis, OR, USA \\ E-mail: Cory.Simon@oregonstate.edu
}

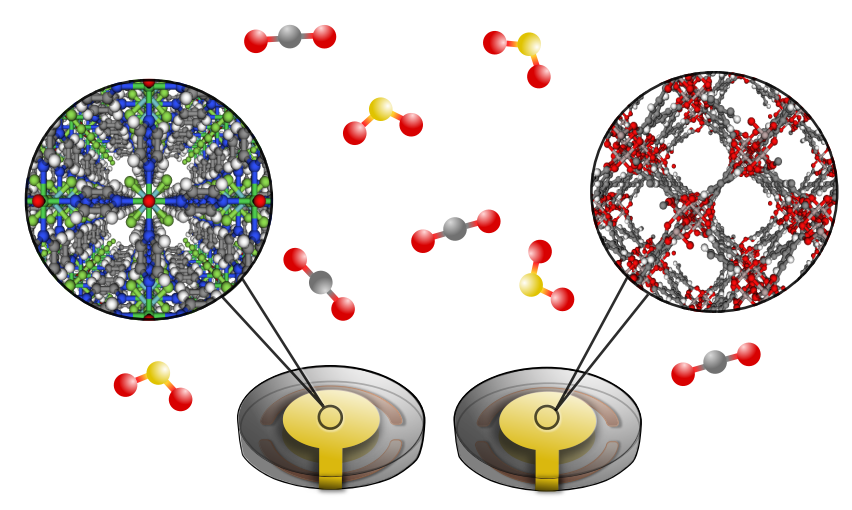

\section{Abstract}

Metal-organic frameworks (MOFs)- tunable, nano-porous materials- are alluring recognition elements for gas sensing. Mimicking human olfaction, an array of cross-sensitive, MOFbased sensors could enable analyte detection in complex, variable gas mixtures containing confounding gas species. Herein, we address the question: given a set of MOF candidates and their adsorption properties, how do we select the optimal subset to compose a sensor array that accurately and robustly predicts the gas composition via monitoring the adsorbed mass in each MOF? We first mathematically formulate the MOF-based sensor array problem under dilute conditions. Instructively, the sensor array can be viewed as a linear map from gas composition space to sensor array response space defined by the matrix $\mathbf{H}$ of Henry coefficients of the gases in the MOFs. Characterizing this mapping, the singular value decomposition of $\mathbf{H}$ is a useful tool for evaluating MOF subsets for sensor arrays, as it determines the sensitivity of the predicted gas composition to measurement error, quantifies the magnitude of the response to changes in composition, and recovers which direction in gas composition space elicits the largest/smallest response. To illustrate, on the basis of experimental adsorption data, we curate MOFs for a sensor array with the objective of determining the concentration of $\mathrm{CO}_{2}$ and $\mathrm{SO}_{2}$ in the gas phase. 


\section{Introduction}

Gas/vapor sensors are crucial to monitor and control industrial processes, ${ }^{1}$ ensure safety, and detect security threats. ${ }^{2}$ New applications for gas sensors are emerging, e.g., in food quality assessment, ${ }^{3}$ urban air quality monitoring, ${ }^{4,5}$ and diagnosis of disease. ${ }^{6}$ For both emerging and existing markets for gas sensors, improvements are needed in the limits of detection, sensitivity and specificity (if classification) or accuracy (if regression) and the robustness thereof to a diversity of environments and confounding/interfering gas species (i.e., mitigating cross-sensitivity), chemical and thermal stability, portability/size, lifetime, response time, power consumption, and cost.

Solid state gas sensors function as follows. Analyte molecules from the gas phase interact (physically or chemically) with a material (the recognition element) and induce a change in its property. A transducer then converts this change in property into a measurable signal, thereby providing information about the concentration of the analyte. As example recognition elements for gas sensors: conducting polymers ${ }^{7,8}$ exhibit a change in electrical resistance when gas molecules adsorb into the polymer matrix and interact with it; metalloporphyrins offer open coordination metal sites that bind gas molecules and change color depending on the identity of the gas species bound; ${ }^{9}$ semiconducting metal oxides ${ }^{10-12}$ exhibit a change in electrical conductivity when gas interacts/reacts with the surface and chemisorbed oxygen on its surface to change the concentration of charge carriers (electrons/holes).

In this work, we consider a gas sensor comprised of a quartz crystal microbalance $(\mathrm{QCM})^{13}$ coated with a nanoporous material thin film, such as a metal-organic framework (MOF) film. ${ }^{14-17}$ As the recognition element, MOFs harbor large internal surface areas ${ }^{18}$ and adsorb (and concentrate) gas molecules discriminately. ${ }^{19}$ Therefore, the total mass of adsorbed gas in the porous material sensitively depends on and therefore provides information about the composition of the gas phase. As for the transducer, QCMs can detect mass changes on the order of nanograms by applying an alternating electrical current to the piezoelectric, quartz crystal and measuring its vibration frequency. The Sauerbrey equation ${ }^{20}$ relates a change in the vibrational frequency of the crystal to a change in mass loading (caused by a change in the amount of gas adsorbed in the MOF) under simplifying assumptions. ${ }^{15} \mathrm{Sev}$ eral studies ${ }^{21-29}$ demonstrate that MOF-coated QCMs can reliably detect changes in composition of the gas phase; though, typically experiments are conducted only with simple (e.g., binary) gas mixtures.

Monitoring the adsorption in a single porous material is likely inadequate for gas sensing in practical applications due to cross-sensitivity. Exposed to a complex gas mixture, such as the breath of a patient, ${ }^{30}$ a porous material will adsorb all constituents of the mixture, although to varying degrees. Because each constituent of the mixture contributes to the measured (total) mass of adsorbed gas in a porous material, we cannot definitively associate mass changes with any analyte in particular ${ }^{1}$. To provide more information and distinguish changes in the concentration of the target analyte from changes in concentrations of confounding/interfering gas species (i.e., to address cross-sensitivity), the key is to monitor the response of an array of different QCM-MOF sensors responding to the same gas mixture. ${ }^{31-33}$ The information-rich response pattern of a sensor array can then be parsed to determine the concentration of the target analyte, teasing out the contribution to the response by interfering/confounding gas species in the mixture to which each material in the sensor array is exposed. Such a set of diverse yet, likely, highly cross-sensitive sensors responding to the same gas mixture is coined an electronic nose (e-nose) $)^{34,35}$ and mimics human olfaction (see Box 1).

\footnotetext{
${ }^{1}$ We are precluding here the unlikely possibility that we can synthesize a MOF that is extremely selective for the analyte and excludes all other gas species, which would enable us to attribute all adsorbed mass changes to changes in the analyte concentration.
} 
Box 1: Mimicking human olfaction

Interestingly and instructively, a sensor array mimics the human olfactory system. Inside our nasal cavities are bipolar, olfactory sensor neurons. The dendrite of these neurons possesses cilia exposed to the airspace. Odorant molecules bind to and activate transmembrane proteins, olfactory receptors, on the cilia. ${ }^{36,37}$ Approximately 400 different ${ }^{38,39}$ olfactory receptors are expressed in human (comparatively, dogs: $>800,{ }^{40}$ flies: ca. $60^{41}$ ), and each olfactory neuron is thought to express only one type of receptor. ${ }^{42,43}$ The axons of the olfactory sensory neurons extend through the skull and signal to the brain, which interprets the response pattern of the olfactory neurons and brings awareness to the aromas around us. Importantly, the olfactory receptors are cross-sensitive: several different odorants bind and active a given olfactory receptor, and each odorant activates several olfactory receptors. ${ }^{42}$ This is analogous to a MOF-based sensor array where, practically, no MOF will exhibit perfect adsorptive selectivity. Yet, despite the crosssensitivity of the olfactory neurons, the human olfactory system can (a) distinguish among a very large ${ }^{44}$ but disputed ${ }^{45,46}$ number of odors and (b) detect trace concentrations of odors; e.g., our detection threshold for hydrogen sulfide is $<1 \mathrm{ppb} .{ }^{47}$ Instructed by our own remarkable olfactory system, we conclude that engineering an accurate sensor for complex, highly variable gas mixtures does not necessitate a set of materials with [impractically] perfect adsorptive selectivity for each constitutive analyte in the mixture, but, rather can be achieved by monitoring the response pattern of a set of cross-sensitive (yet diverse) materials. ${ }^{31,48}$ Analogous to the brain, we need a (computational) pattern recognition algorithm to interpret the high-dimensional signal produced by the sensors, e.g. an artificial neural network, principal component analysis, linear discriminant analysis, etc. ${ }^{35,49,50}$ See Refs. ${ }^{51,52}$ for reviews on mathemati$\mathrm{cal} /$ statistical methods to parse a sensor array response.
MOFs have garnered much attention for use as recognition elements in gas/vapor sensors $^{16,17,53-55}$ for several reasons. (1) MOFs offer large internal surface areas and extensive porosity ${ }^{18}$ for selectively concentrating analytes in their pores and eliciting a sensitive response to composition changes in the gas phase. (2) The diversity of pore geometries and internal surface chemistries among the tens of thousands of MOFs synthesized ${ }^{56}$ enables the curation of a diverse set of materials to comprise a sensor array that yields an information-rich response pattern. The tunability of MOFs stems from their modular synthesis, where metal nodes or clusters and organic linkers self-assemble to form a crystalline framework. ${ }^{57}$ (3) MOFs often exhibit high thermal and chemical stability. ${ }^{58}$ (4) Sensors based on reversible physical adsorption are reusable, as opposed to singleuse. (5) The crystalline nature of MOFs facilitates their reproducible fabrication. (6) MOFs simplify portable sensing devices by combining concentration and detection of the analyte in one step, as opposed to other analytic methods that first pre-concentrate the analyte. ${ }^{59-61}$ (7) As opposed to e.g., metal oxide sensors ${ }^{10-12}$ that operate at elevated temperatures, MOFs can operate at room temperature. (8) Intriguingly, coordinatively unsaturated (open) metal sites in $\mathrm{MOFs}^{62}$ are a striking analogy to the open metal sites thought to bind odorants in mammalian olfactory receptors. ${ }^{63}$ (9) We can use molecular models and simulations to computationally predict the adsorption properties of MOFs, ${ }^{64}$ then, on the basis of these simulated adsorption properties, judiciously curate a set of MOFs for an application-specific sensor array based on adsorbed mass changes. ${ }^{65,66}$ As candidates, databases of hundreds of thousands of experimental and theoretical MOF structures have been curated. ${ }^{56,67,68}$

Gustafson and Wilmer ${ }^{65,66}$ pioneered the computational selection of MOFs for sensor arrays on the basis of predicted mixed-gas adsorption via molecular simulations. First, they posed a sensor array score that characterizes the norm of the sensor array response when the gas phase composition changes, quantifying the ability of the response to distinguish between 
two gas mixtures. ${ }^{65}$ Importantly, Gustafson and Wilmer found that a different set of MOFs is optimal depending on the pressure of the mixture; i.e., the optimal set of MOFs depends on the operating condition. ${ }^{65}$ Second, Gustafson and Wilmer ${ }^{66}$ accounted for experimental measurement error by adding noise to simulated adsorption measurements in each MOF within a sensor array, then using the simulated sensor array response to predict the composition of the (known) gas mixture. They quantified the information content in the sensor array response by computing the Kullback-Liebler divergence between the distribution of predicted gas compositions and a uniform distribution over composition space. Recently, they showed that a genetic algorithm can efficiently select MOFs for very large sensor arrays where the number of possible combinations of MOF candidates is prohibitive for brute-force screening. ${ }^{69}$

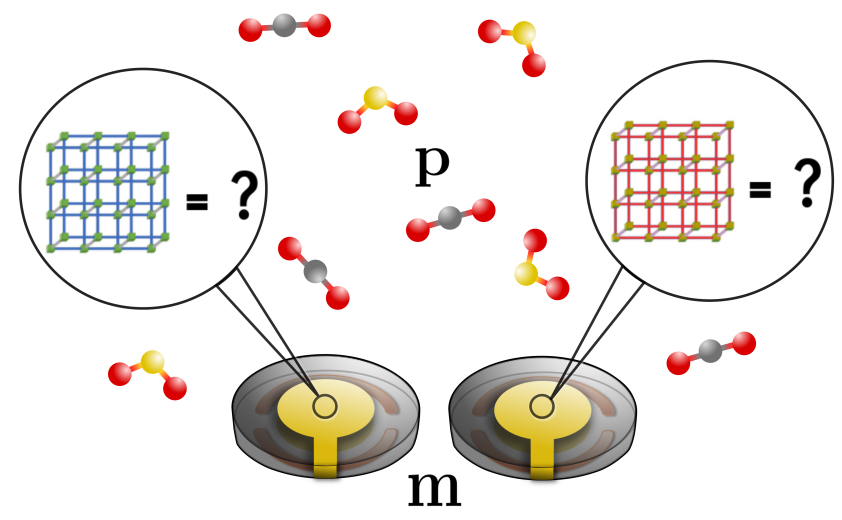

Figure 1: A schematic of a sensor array composed of two QCMs coated with thin films of two different MOFs. The goal is to predict the gas phase composition $\mathbf{p}$ from the sensor array response $\mathbf{m}$, which consists of the adsorbed mass measurements by the QCMs. Herein, we explore how to, given a set of MOF candidates and their adsorption properties, choose the optimal subset of MOFs for a QCM-MOF sensor array to accurately determine the gas composition in the face of measurement error.

The central question we address herein is (see Fig. 1): given a set of candidate MOFs and their (experimental or predicted) adsorption properties, how do we choose the optimal subset of MOFs to constitute a QCM-MOF sen- sor array that accurately and robustly predicts the composition of the gas phase in the face of measurement error? Measurement error corrupts the prediction of the gas composition by the sensor array and emanates from e.g., (a) adsorbed mass measurements by the QCMs and (b) estimates of the parameters in the gas adsorption model. We consider dilute conditions, where adsorption is governed by Henry's law. Although restrictive, this lends the system to be instructively viewed as a linear transformation from gas composition space to sensor array response space. ${ }^{70}$ We show that the singular value decomposition ${ }^{71}$ of the matrix of Henry coefficients of the gases in the MOFs in the sensor array (i) determines the sensitivity of the predicted gas composition to measurement error, (ii) quantifies the magnitude of the sensory array response to changes in the gas composition, and (iii) reveals the anisotropic nature of the sensor array response and recovers which direction in composition space elicits the largest/smallest array response. Therefore, we can assess the performance of a hypothetical QCM-MOF sensor array with the singular value decomposition of the matrix of Henry coefficients of the different gases in the candidate MOFs; this serves as a screening tool for choosing a subset of MOFs among a large number of candidates for a QCM-MOF sensor array on the basis of experimental or simulated adsorption properties. While valid only at dilute conditions, this framework can be extended outside the dilute regime, accounting for saturating adsorption, via a linearized model approximately valid in a neighborhood surrounding a baseline gas composition. Finally, we outline mathematical arguments that adding an additional QCM$\mathrm{MOF}$ to an incumbent sensor array, under some assumptions, can only improve the accuracy of the prediction of the gas composition.

\section{Framing the QCM-MOF sensor array problem}




\subsection{Adsorption model for a sen- sor array in dilute conditions}

We first pose an adsorption model, valid in dilute conditions, for a sensor array composed of $n$ QCM-MOF sensors responding to a gas phase with $g$ constituents.

QCM $i$ will measure the total mass of gas adsorbed- with contributions from each of the $g$ gases- on the MOF thin film that coats it, $m_{i}$ [g gas]. Under Henry's law, the contributions of each gas species to adsorption are additive and linear in partial pressure:

$$
m_{i}=H_{i, 1} p_{1}+H_{i, 2} p_{2}+\cdots+H_{i, g} p_{g},
$$

where $H_{i, j}$ is the Henry coefficient [g gas/bar] of gas $j$ in the MOF coating QCM $i$ and $p_{j}$ is the partial pressure [bar] of gas $j$, felt by each MOF in the sensor array. Henry's law in eqn. 1 is valid only in dilute conditions and assumes that the adsorbed gas molecules do not interact (attraction/repulsion) with each other. ${ }^{72}$

In vector form, the adsorption model for an array of $n$ QCM-MOF sensors responding to a gas phase with $g$ constituents is:

$$
\mathbf{m}=\mathbf{H p},
$$

where $\mathbf{m} \in \mathbb{R}^{n}$ is the vector of QCM mass measurements, $\mathbf{p} \in \mathbb{R}^{g}$ is the vector of partial pressures of the gases, and $\mathbf{H}$ is the $n \times g$ Henry coefficient matrix whose element $(i, j)$ is the Henry coefficient of gas $j$ in MOF $i\left(H_{i, j}\right.$ in eqn. 1).

For convenience, we introduce deviation variables $\mathbf{p}^{*}$ and $\mathbf{m}^{*}$ to describe changes in the gas composition and sensor array response, respectively, from a nominal baseline, "business-asusual" gas composition $\overline{\mathbf{p}}$ and its associated response $\overline{\mathbf{m}}$ :

$$
\begin{aligned}
\mathbf{p}^{*} & :=\mathbf{p}-\overline{\mathbf{p}} \\
\mathbf{m}^{*} & :=\mathbf{m}-\overline{\mathbf{m}}
\end{aligned}
$$

Since $\overline{\mathbf{m}}=\mathbf{H} \overline{\mathbf{p}}$ by definition of $\overline{\mathbf{m}}$, eqn. 2 expressed in deviation form is:

$$
\mathbf{m}^{*}=\mathbf{H p}^{*} .
$$

The change in the sensor array response now follows from a change in the gas composition from baseline conditions, with the Henry coefficient matrix $\mathbf{H}$ unchanged. We restrict $\overline{\mathbf{p}}$ to lay in the Henry regime of each MOF.

Note that eqn. 5 neglects the dynamics associated with ad/desorption, takes the gas phase as in thermodynamic equilibrium with the adsorbed phase, and assumes isothermal conditions.

\subsection{The sensor array computa- tion under dilute conditions}

A sensor array for $g$ gases comprised of $n$ QCMMOFs operates in dilute conditions as follows. First, as calibration, we measure the Henry coefficients of each of the $g$ gases in each of the $n$ MOFs to construct the Henry coefficient matrix, H. Then, during sensor array deployment, we observe the mass measurements by the QCMs, $\mathbf{m}$. On the basis of $\mathbf{H}$ and $\mathbf{m}$, we then use eqn. 2 to predict the gas phase composition p. Our goal to quantitatively predict the gas composition $\mathbf{p}$ is distinct from an alternative formulation of gas sensing as a classification; see Box 2.

\section{Box 2: Classifying distinctive mix-}

tures

A goal of a gas sensor array could also be to discriminate between complex and distinctive gas mixtures (classification) whose constituents are not necessarily known. For example, the response of a sensor array to human breath could distinguish healthy from ill patients; ${ }^{73}$ signatures of volatile organic compounds in the breath are biomarkers for lung ${ }^{74}$ and colon cancer. ${ }^{75}$ Here, the goal is not necessarily to directly identify the constituents in the breath that distinguish healthy from ill patients (which could be daunting), but merely to build a sensor array whose response contains enough information to distinguish healthy from ill patients as a diagnostic tool. Conceptually, within the high-dimensional response space of the sensor array, the desired outcome is for the responses to the breath of healthy and can- 


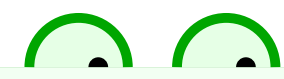

cer patients to congregate in disparate regions, separated by a (learnable) manifold embedded in this high-dimensional space. Without incorporating knowledge about the compounds in the breath that distinguish healthy from cancer patients, Machado et al. ${ }^{76}$ constructed a sensor array of 32 polymer composites and discriminated between the breath of patients with and without lung cancer, and Peng et al. constructed a sensor array of 14 cross-sensitive, organically functionalized gold nanoparticles and discriminated between patients with and without colon cancer. ${ }^{75}$ Such an aim to classify sensor array responses contrasts to the goal in this work of predicting the concentration of a set of known analytes in the gas phase (regression).

\subsubsection{Case $n<g$ : underdetermined}

The response of a sensor array composed of fewer sensors than gas phase constituents $(n<$ g) contains insufficient information to arrive at a unique prediction of the gas composition, $\mathbf{p}$. Mathematically, $\mathbf{m}=\mathbf{H p}$ is underdetermined; infinitely many solutions $\mathbf{p}$ exist $^{2}$, but $\mathbf{p}$ is constrained to lie in a subspace of gas composition space. Clearly, we need as many sensors as gas phase constituents (whose partial pressures are unknown) to determine $\mathbf{p}$.

\subsubsection{Case $n=g$ : determined}

If $n=g$ and the MOFs coating the QCM are distinct, $\mathbf{H}^{-1}$ exists, and the predicted gas composition $\mathbf{p}$ is uniquely determined from eqn. 2 as $\mathbf{p}=\mathbf{H}^{-1} \mathbf{m}$. This excludes the improbable outcome (under the constraint of the MOFs being unique) that $\mathbf{H}$ has linearly dependent rows or columns to render it singular.

\footnotetext{
${ }^{2}$ While pathological, an underdetermined system could also be inconsistent, where no solution exists. This outcome occurs in a two-sensor array with $g>2$, where two identical MOFs are used, giving two duplicate rows in $\mathbf{H}$; if the QCMs give two different adsorbed mass measurements due to measurement error, the two Henry law equations are inconsistent.
}

\subsubsection{Case $n>g$ : overdetermined}

If $n>g$ and the MOFs coating the QCM are distinct, $\mathbf{m}=\mathbf{H p}$ is overdetermined; an exact solution for $\mathbf{p}$ does not exist. This excludes the highly improbable outcome that zero error emanates from the measurements and model such that $\mathbf{m}$ lies in the column space of $\mathbf{H}$; i.e., practically, measurement and model error preclude exact satisfaction of all $n$ equations. However, we can find an approximate solution $\mathbf{p}^{+}$to $\mathbf{m}=\mathbf{H p}$ for an overdetermined system; we predict the gas composition by minimizing the norm of the error, $\mathbf{e}(\tilde{\mathbf{p}}):=\mathbf{m}-\mathbf{H} \tilde{\mathbf{p}}$ :

$$
\mathbf{p}^{+}:=\underset{\tilde{\mathbf{p}}}{\arg \min }\|\mathbf{m}-\mathbf{H} \tilde{\mathbf{p}}\|^{2} .
$$

The solution to this least squares optimization problem (see Sec. S4) satisfies the so-called normal equation:

$$
\mathbf{H}^{\top} \mathbf{H p}^{+}=\mathbf{H}^{\top} \mathbf{m} .
$$

Provided that $\mathbf{H}$ has independent columns, a unique solution to eqn 7 exists, $\mathbf{p}^{+}=\mathbf{H}^{+} \mathbf{m}$, where the matrix $\mathbf{H}^{+}:=\left(\mathbf{H}^{\top} \mathbf{H}\right)^{-1} \mathbf{H}^{\top}$ is the left inverse of $\mathbf{H}$. The matrix $\mathbf{H H}^{+}$projects $\mathbf{m}$ onto the column space of $\mathbf{H}$.

\subsection{View as a linear map}

Eqn. 5 provides a useful geometric perspective: an array of $n$ QCM-MOF sensors responding to the gas phase with $g$ constituents is a linear mapping of a vector $\mathbf{p}^{*} \in \mathbb{R}^{g}$ in gas composition space to a vector $\mathbf{m}^{*} \in \mathbb{R}^{n}$ in sensor array response space. ${ }^{70}$ This linear mapping $\mathbf{p}^{*} \mapsto \mathbf{m}^{*}$ is defined by the $n \times g$ Henry coefficient matrix $\mathbf{H}$, i.e., by the adsorption properties of the MOFs, and will aid our judicious curation of MOFs for a sensor array. Before parsing Fig. 2 that displays visualizations of this linear map under different scenarios, we introduce the singular value decomposition that helps us parse this mapping. 


\subsubsection{The singular value decomposition}

The singular value decomposition (SVD) ${ }^{71,77}$ of the $n \times g$ Henry coefficient matrix $\mathbf{H}$ is:

$$
\mathbf{H}=\mathbf{U} \boldsymbol{\Sigma} \mathbf{V}^{\top},
$$

where $\mathbf{U}$ is an $n \times n$ orthogonal matrix whose columns are the left singular vectors that lie in sensor array response space, $\mathbf{V}$ is a $g \times g$ orthogonal matrix whose columns are the right singular vectors that lie in gas composition space, and $\Sigma$ is an $n \times g$ diagonal matrix containing the singular values $\sigma_{1} \geq \sigma_{2} \geq \cdots \geq \sigma_{\min (n, g)}$ of $\mathbf{H}$ running down its diagonal in non-increasing order. Seen by $\mathbf{H V}=\mathbf{U} \boldsymbol{\Sigma}$, right singular vector $\mathbf{v}_{i}$ is mapped to left singular vector $\mathbf{u}_{i}$ scaled by its cognate singular value $\sigma_{i}$, for $i \in\{1,2, \ldots, k\}$, while $\mathbf{v}_{i}$ for $i \in\{k+1, k+2, \ldots, g\}$ lie in the null space of $\mathbf{H}$, given that $k$ is the rank of $\mathbf{H}$ (implying $\left.\sigma_{k+1}=\sigma_{k+2}=\cdots=\sigma_{\min (n, g)}=0\right)$.

The SVD of $\mathbf{H}$ elucidates the mapping of a unit $(g-1)$-sphere $\left(\left\{\mathbf{p}^{*} \in \mathbb{R}^{g}:\left\|\mathbf{p}^{*}\right\|=1\right\}\right)$ in gas composition space into sensor response space as a sequence of three transformations: $\mathbf{H p}^{*}=\mathbf{U} \boldsymbol{\Sigma} \mathbf{V}^{\top} \mathbf{p}^{*}$. The $(g-1)$-sphere is first rotated/reflected by the orthogonal matrix $\mathbf{V}^{\top}$. Next, it is embedded $(n>g)$ or collapsed $(n<g)$ into the $n$-dimensional sensor response space and stretched/compressed along the coordinate axes by $\boldsymbol{\Sigma}$, with the singular values as scaling factors. A zero singular value results in the collapse of a dimension, so this ellipsoid lies in a $k$-dimensional subspace of sensor array response space (where $k$ is the rank of $\mathbf{H})$. Finally, the ellipsoid is rotated/reflected by the orthogonal matrix $\mathbf{U}$. The $k$ principal semi-axes of the resulting ellipsoid in sensor response space point in the direction $\mathbf{u}_{i}$ and are of magnitude $\sigma_{i}$. In summary, the SVD shows that the image of a unit $(g-1)$-sphere in gas composition space under rank $k$ matrix $\mathbf{H}$ is (if $\mathbf{H}$ is full column rank, the surface of) an ellipsoid embedded in the $n$-dimensional sensor array response space. Right singular vector $\mathbf{v}_{i}$ (on the unit $(g-1)$-sphere) in gas composition space is mapped to the ellipsoid's principal semi-axis $\sigma_{i} \mathbf{u}_{i}$ in sensor array response space for $i \in\{1,2, \ldots, k\}$. The index of the singular value ranks the ellipsoid's semi-axes by length.
Understood by this sequence of linear maps, the SVD of $\mathbf{H}$ reveals the largest and smallest norm of the sensor array response $\mathbf{m}^{*}$ among all $\mathbf{p}^{*}$ laying on a $(g-1)$-sphere:

$$
\begin{aligned}
\|\mathbf{H}\|_{o p}:= & \max _{\mathbf{p}^{*} \neq \mathbf{0}} \frac{\left\|\mathbf{H} \mathbf{p}^{*}\right\|}{\left\|\mathbf{p}^{*}\right\|}=\sigma_{1} \\
& \min _{\mathbf{p}^{*} \neq \mathbf{0}} \frac{\left\|\mathbf{H} \mathbf{p}^{*}\right\|}{\left\|\mathbf{p}^{*}\right\|}=\sigma_{\min (n, g)} .
\end{aligned}
$$

The first relation is defined as the operator norm of the matrix $\mathbf{H}$. The maximum and minimum factors by which a vector's norm can be scaled upon being transformed by $\mathbf{H}$ is the largest and smallest singular value, respectively. The singular values of $\mathbf{H}$ are central to evaluating a set of candidate MOFs for a sensor array because they determine the maximum and minimum norm of the sensor array response under changes in gas composition of a given magnitude.

\subsubsection{Caricatures of linear maps $\mathrm{p}^{*} \mapsto$ $\mathrm{m}^{*}$}

To gain insights for sensor array design, we next visualize how a circle in $g=2$-dimensional gas composition space is mapped to sensor array response space for $n \in\{1,2,3\}$ sensors. See Fig. 2 for caricatures we discuss below. Clearly, the sensor array response is anisotropic: though exposed to gas composition changes of the same magnitude, the magnitude of the sensor array response depends on the direction moved in composition space.

Fig. 2(a): one sensor [underdetermined]. One sensor rotates the circle in gas composition space, collapses it into a line segment in the 1D sensor response space, then stretches or compresses it. This mapping is not invertible, as two gas compositions are mapped to the same sensor response (see color). The second right singular vector $\mathbf{v}_{2}$ is mapped to the zero vector; a gas composition change in this direction does not elicit a response because the adsorption of one species equates the desorption of the other species. 


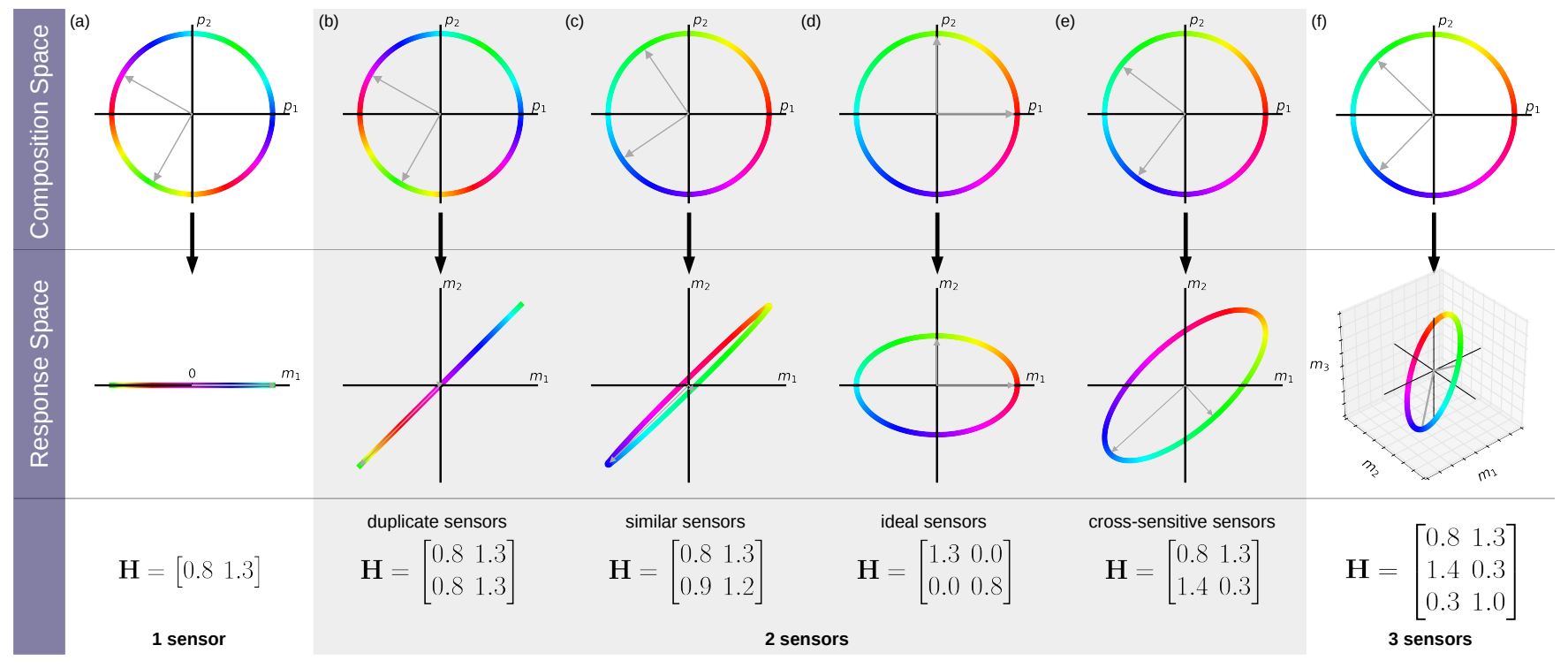

Figure 2: The view of a sensor array as a linear map $\mathbf{p}^{*} \mapsto \mathbf{m}^{*}$ from gas composition space (top) to sensor array response space (middle) via the Henry coefficient matrix $\mathbf{H}$ (bottom); see eqn. 5. Caricatures of the mapping of a circle in a $g=2$-component gas composition space to an ellipse/ellipsoid in sensor array response space for (a) $n=1$ sensor, (b-e) $n=2$ sensors, (f) $n=3$ sensors. A point of a given color in composition space is mapped to the same color in sensor response space. Gray arrows in composition and response space, respectively, denote $\mathbf{v}_{i}$ and $\sigma_{i} \mathbf{u}_{i}$ from the singular value decomposition of $\mathbf{H}$.

Fig. 2(b): two duplicate sensors [underdetermined]. Consider a sensor array composed of two duplicate sensors and neglect measurement error in $\mathbf{m}$. Such a sensor array response is inherently 1D: the circle in gas composition space is rotated, collapsed to a line segment and stretched or compressed, then rotated again, remaining in a $2 \mathrm{D}$ space throughout. The line segment makes a $\pi / 4$ angle with the $m_{1}$-axis because the two MOFs ad/desorb an identical amount of gas at all compositions. Again, (i) the mapping is not invertible; two compositions map to the same response and (ii) the second right singular vector $\mathbf{v}_{2}$ is mapped to the zero vector, where adsorption of one gas species equates desorption of the other species. Both are a consequence of the two duplicate rows in $\mathbf{H}$ that render it singular $\left(\sigma_{2}=0\right)$.

Fig. 2(c): two similar sensors [determined]. Rather than coating two QCMs with an identical MOF, suppose instead we graft a functional group on the internal surface of the pore in one the MOFs, resulting in two MOFs that are not identical but differ only marginally.
Owing to structural similarity, we expect the Henry coefficient matrix for such a sensor array to take the form:

$$
\mathbf{H}=\left[\begin{array}{cc}
H_{1,1} & H_{1,2} \\
H_{1,1}+\epsilon & H_{1,2}+\delta
\end{array}\right],
$$

where the Henry coefficients of the two gases in the two MOFs deviate by small, non-zero numbers $\epsilon$ and $\delta$. Though $\mathbf{H}$ in eqn. 11 is now likely invertible, its rows point in a similar direction, rendering it, colloquially, "almost singular".

The image of a circle in composition space by such a sensor array is a highly eccentric ellipse in sensor array response space. A change in composition in the direction of $\mathbf{v}_{1}$ elicits a much larger sensor response than in the direction of $\mathbf{v}_{2}$; the response is highly anisotropic. The semi-major axis of the ellipse makes a near$\pi / 4$ angle with the $m_{1}$-axis because the mass of gas adsorbed by each of the two MOFs is similar in all gas mixtures. The mapping in Fig. 2(c) is one-to-one (see color), in contrast to the case of duplicate sensors, yet differs only marginally from Fig. 2(b), reinforcing that two similar sensors lead to an "almost singular" sys- 
tem $\mathbf{m}=\mathbf{H p}$

Fig. 2(d): two diverse sensors, ideal [determined]. In an ideal sensor array, MOF $i$ is perfectly selective for gas $i$, for $i \in\{1,2, \ldots, g\}$. Sensor $i$ in the array can then be independently analyzed to determine the concentration of gas phase constituent $i$. An ideal sensor array maps a circle in gas composition space to an ellipse in sensor response space whose principal axes are aligned with the coordinate axes. The left and right singular vectors are all in the direction of the coordinate axes. The response could still be anisotropic, however, depending on how the Henry coefficients $H_{i, i}$ compare.

Fig. 2(e): two diverse sensors, crosssensitive [determined]. Practically, each MOF will exhibit cross-sensitivity and adsorb all gases to some extent. In this case, the response of any single sensor within the array is insufficient to determine the concentration of any particular gas phase constituent; the response $\mathbf{m}$ as a whole must be analyzed to determine the concentration of any particular gas phase constituent. Under a cross-sensitive sensor array with two diverse MOFs that each adsorb both gas constituents appreciably, the image of a circle differs from the ideal sensor array in that both of the following are not aligned with the coordinate axes: (i) the principal axes of the ellipse in sensor response space (left singular vectors) and (ii) the directions in gas composition space that elicit the largest and smallest sensor array response (right singular vectors).

Fig. 2(f): three diverse sensors [overdetermined]. Finally, consider the linear map under a sensor array composed of three diverse, cross-sensitive MOFs. The circle in composition space is rotated, embedded into the $3 \mathrm{D}$ sensor array response space, stretched or compressed, then rotated again in the $3 \mathrm{D}$ sensor response space. The mapping is one-to-one (see color), but, if the sensor array response $\mathbf{m}^{*}$ does not lie on the same plane as the ellipse- i.e., if $\mathbf{m}^{*}$ is not in the column space of $\mathbf{H}$ - there is no solution to eqn. 5 since no point in composition space is mapped to that response $\mathbf{m}^{*}$. Owing to measurement and model error, this is the practical outcome, and we resort to eqn. 6 to project $\mathbf{m}^{*}$ onto the plane on which the ellipse in Fig. 2(f) lies and arrive at a prediction of the composition, $\mathbf{p}^{+}$.

\subsection{The optimal, robust sensor array}

Given that $\mathbf{m}=\mathbf{H p}$ is not underdetermined, it is not immediately apparent that one set of MOFs is more suitable than another-i.e., that one Henry coefficient matrix $\mathbf{H}$ is better than another- for determining $\mathbf{p}$ from the array response m. Though, comparison of Figs. 2(b) and (c) suggests that the sensor array composed of two similar sensors differs only marginally from the duplicate sensor case (underdetermined). We now show that acknowledging measurement error is the key to understanding how to curate MOFs for robust and accurate sensor arrays. Measurement error, which corrupts our estimate of the gas composition from $\mathbf{m}=\mathbf{H p}$, emanates from two sources: the adsorbed mass measurements by the QCMs and our measurements of the Henry coefficients of the gases in the MOFs. We outline two disparate perspectives below that lead to the following tool to evaluate subsets of MOFs for sensor arrays in dilute conditions: the subset of MOFs with which to coat QCMs to compose the most robust and accurate sensor array under measurement error is the one whose Henry coefficient matrix $\mathbf{H}$ has the largest smallest singular value (i.e., the largest $\sigma_{g}(\mathbf{H})$, assuming $n \geq g$ ).

\subsubsection{A linear map perspective}

Consider when the mass measurements by the QCMs are corrupted by error and we aim to simply determine from $\mathbf{m}^{*}$ whether the gas composition has moved a distance from baseline conditions $\overline{\mathbf{p}}$ or not (i.e., if $\left\|\mathbf{p}^{*}\right\|>r$ with $r$ some threshold distance e.g., representing an exposure limit). Under normally distributed measurement error, we are not confi- 
dent enough to distinguish a sensor array response of small magnitude from a $\mathbf{m}^{*}=\mathbf{0}$ response corresponding to $\mathbf{p}=\overline{\mathbf{p}}$. From the perspective of the linear maps in Fig. 2, then, we seek a set of MOFs that elicit a response to $\left\{\mathbf{p}^{*}:\left\|\mathbf{p}^{*}\right\|=r\right\}$ large enough to distinguish from measurement error (assumed not to scale with $\left.\left\|\mathbf{m}^{*}\right\|\right)$. Therefore, according to eqn. 10, we desire the smallest singular value of $\mathbf{H}$ to be large to guarantee that the norm of the sensor array response $\left\|\mathbf{m}^{*}\right\|=\left\|\mathbf{H p}^{*}\right\|$ will be large so as to be distinguishable from error- regardless of the direction moved in gas composition space (robustness). For example, the map $\mathbf{p}^{*} \mapsto \mathbf{m}^{*}$ under the sensor array composed of similar sensors in Fig. 2(c)- where $\mathbf{H}$ was "almost singular" - indicates that the sensor array response is very small and, under measurement error, indistinguishable from zero if the gas changes composition in the direction of $\mathbf{v}_{2}$. Relating to the mapping perspective in Sec. 2.3.1: ensuring that $\sigma_{g}(\mathbf{H})$ is large guarantees the image of the $(g-1)$-sphere in composition space, which is the surface of an ellipsoid provided $\mathbf{H}$ is full column rank, is sufficiently far from the origin so as to distinguish from measurement error the responses to all gas compositions on the $(g-1)$-sphere.

\subsubsection{A conditioning perspective}

We now consider the conditioning of $\mathbf{m}=\mathbf{H p}$, i.e., how much the predicted gas composition changes upon small changes in the measured adsorbed masses by the QCMs or Henry coefficients. We aim to choose a subset of MOFs for a sensor array such that the solution to $\mathbf{m}=\mathbf{H p}$ is insensitive to small perturbations in $\mathbf{H}$ and $\mathbf{m}$. We show this aim is met by choosing the subset of MOFs whose $\mathbf{H}$ possesses the largest smallest singular value.

Consider a small perturbation in the estimated Henry coefficients $\mathbf{H}$ and adsorbed mass measurements $\mathbf{m}$ by the QCMs, $\delta \mathbf{H}$ (an $n \times g$ matrix) and $\delta \mathbf{m} \in \mathbb{R}^{n}$, respectively. These perturbations result in a change in the predicted gas phase composition, $\delta \mathbf{p} \in \mathbb{R}^{g}$, which we desire to be of small magnitude ${ }^{3}$. Because $\mathbf{p} \mapsto \mathbf{m}$

\footnotetext{
${ }^{3}$ We assume that we value an accurate estimation of
}

is generally anisotropic, $\delta \mathbf{p}$ depends on the direction we move in composition space, but we will seek an upper bound on its norm for a robust sensor array.

Determined systems, $n=g$. For a determined system, the perturbed problem is:

$$
\mathbf{m}+\delta \mathbf{m}=(\mathbf{H}+\delta \mathbf{H})(\mathbf{p}+\delta \mathbf{p})
$$

where $\mathbf{m}+\delta \mathbf{m}$ is the perturbed measurement of the mass adsorbed in each MOF thin film by the QCMs and $\mathbf{H}+\delta \mathbf{H}$ is the perturbed Henry coefficient matrix; $\mathbf{p}+\delta \mathbf{p}$ is the predicted gas composition under these perturbations, which differs from $\mathbf{p}=\mathbf{H}^{-1} \mathbf{m}$ in the unperturbed problem by $\delta \mathbf{p}$ by definition. We seek an upper bound on $\|\delta \mathbf{p}\|$. Taking existence of $\mathbf{H}^{-1}$, we solve eqn. 12 for $\delta \mathbf{p}$ after neglecting the second order error term and noting $\mathbf{m}=\mathbf{H p}$ is the unperturbed problem:

$$
\delta \mathbf{p}=\mathbf{H}^{-1}(\delta \mathbf{m}-(\delta \mathbf{H}) \mathbf{p}) .
$$

Applying the triangle inequality to eqn. 13 and using definition of the operator norm, we arrive at:

$$
\|\delta \mathbf{p}\| \leq\left\|\mathbf{H}^{-1}\right\|_{o p}\left(\|\delta \mathbf{m}\|+\|\delta \mathbf{H}\|_{o p}\|\mathbf{p}\|\right)
$$

Therefore, to guarantee that $\|\delta \mathbf{p}\|$ is small, we require the operator norm of $\mathbf{H}^{-1}$ to be small. In terms of the SVD of $\mathbf{H}$ in eqn. 8, $\mathbf{H}^{-1}=\mathbf{V} \boldsymbol{\Sigma}^{-1} \mathbf{U}^{\top}$; therefore, the operator norm of $\mathbf{H}^{-1}$ is the inverse of the smallest singular value of $\mathbf{H}$, i.e., $\left\|\mathbf{H}^{-1}\right\|_{o p}=1 / \sigma_{n}(\mathbf{H})$. We conclude that a robust sensor array insensitive to small changes in measured Henry coefficients and adsorbed masses by the QCMs will possess a Henry coefficient matrix $\mathbf{H}$ whose smallest singular value is large.

Overdetermined systems, $n>g$. For an overdetermined system, we resort to least squares optimization in eqn. 6 to estimate the gas composition. The perturbed normal equa-

all $g$ gases equally. 
tion (see eqn. 7) is:

$$
(\mathbf{H}+\delta \mathbf{H})^{\top}(\mathbf{H}+\delta \mathbf{H})(\mathbf{p}+\delta \mathbf{p})=(\mathbf{H}+\delta \mathbf{H})^{\top}(\mathbf{m}+\delta \mathbf{m}) .
$$

Neglecting higher order error terms and noting $\mathbf{H}^{\top} \mathbf{H p}=\mathbf{H}^{\top} \mathbf{m}$ is our unperturbed normal equation, we solve eqn. 15 for $\delta \mathbf{p}$ assuming that $\mathbf{H}$ has independent columns so that its left inverse $\mathbf{H}^{+}$exists:

$$
\delta \mathbf{p}=\mathbf{H}^{+}(\delta \mathbf{m}-(\delta \mathbf{H}) \mathbf{p})+\left(\mathbf{H}^{\top} \mathbf{H}\right)^{-1}(\delta \mathbf{H})^{\top} \mathbf{e} .
$$

Eqn. 16 is analogous to eqn. 13 for determined systems, but with an additional term involving the error $\mathbf{e}:=\mathbf{m}-\mathbf{H p}$ after solving for the least squares solution $\mathbf{p}$ in the unperturbed problem. Applying the triangle inequality and the definition of the operator norm, we arrive at:

$$
\begin{array}{r}
\|\delta \mathbf{p}\| \leq\left\|\mathbf{H}^{+}\right\|_{o p}\left(\|\delta \mathbf{m}\|+\|\delta \mathbf{H}\|_{o p}\|\mathbf{p}\|\right)+ \\
\left\|\left(\mathbf{H}^{\top} \mathbf{H}\right)^{-1}\right\|_{o p}\left\|(\delta \mathbf{H})^{\top}\right\|_{o p}\|\mathbf{e}\| .
\end{array}
$$

Again, we find that the smallest singular value of $\mathbf{H}, \sigma_{g}(\mathbf{H})$, plays a determining role in the upper bound on $\|\delta \mathbf{p}\|$. In terms of the SVD of $\mathbf{H}$ in eqn. 8, the left inverse of $\mathbf{H}$ is:

$$
\mathbf{H}^{+}=\left(\mathbf{H}^{\top} \mathbf{H}\right)^{-1} \mathbf{H}^{\top}=\mathbf{V} \boldsymbol{\Sigma}^{+} \mathbf{U}^{\top},
$$

where $\boldsymbol{\Sigma}^{+}$is a $g$ by $n$ matrix constructed by transposing $\boldsymbol{\Sigma}$ and replacing each (nonzero) singular value on its diagonal with its reciprocal. Therefore, we see $\left\|\mathbf{H}^{+}\right\|_{o p}=1 / \sigma_{g}(\mathbf{H})$. We can also write $\left(\mathbf{H}^{\top} \mathbf{H}\right)^{-1}$ in terms of the SVD of $\mathbf{H}$, revealing that $\left\|\left(\mathbf{H}^{\top} \mathbf{H}\right)^{-1}\right\|_{o p}=1 /\left[\sigma_{g}(\mathbf{H})\right]^{2}$. We conclude from eqn. 17 , for overdetermined systems, the predicted gas composition is insensitive to small changes in the Henry coefficients and adsorbed mass measurements by the QCMs if the smallest singular value of $\mathbf{H}, \sigma_{g}(\mathbf{H})$, is large. Compared to the bound on $\|\delta \mathbf{p}\|$ for determined systems in eqn. 17, overdetermined systems have an extra term arising from the error $\|\mathbf{e}\|$, which scales with the square of the inverse of the smallest singular value of $\mathbf{H}$.

The bounds on $\|\delta \mathbf{p}\|$ in both eqns. 14 and 17 account for two independent ways the sensor array problem $\mathbf{m}=\mathbf{H p}$ can be perturbed: through measurements of (i) adsorbed mass by the QCMs during deployment and (ii) the Henry coefficients of the gases in the MOFs during calibration. For both sources of measurement error, $\sigma_{g}(\mathbf{H})$ determines the sensitivity of the predicted gas composition to perturbations in both $\mathbf{m}$ and $\mathbf{H}$; regardless of which source of measurement error dominates, a large $\sigma_{g}(\mathbf{H})$ mitigates the propagation of perturbations in the measurements to the predicted gas composition.

\section{Demonstration: curating MOFs for $\mathrm{CO}_{2} / \mathrm{SO}_{2}$ sens- ing}

Having framed the QCM-MOF sensor array problem under dilute conditions, we now provide a pedagogical demonstration of the selection of the optimal subset of MOFs to coat QCMs to compose a sensor array for carbon dioxide and sulfur dioxide sensing. $\mathrm{CO}_{2}$ and $\mathrm{SO}_{2}$ are released from human endeavours, such as fossil fuel combustion, ${ }^{78,79}$ as well as from natural occurrences, mainly volcanic eruptions ${ }^{78,80}$ (see Box 3). High atmospheric $\mathrm{SO}_{2}$ concentration causes potentially fatal respiratory diseases ${ }^{81}$ and adversely affects the environment via acid deposition. ${ }^{82}$ While not as toxic as $\mathrm{SO}_{2}$, exposure to high concentrations of $\mathrm{CO}_{2}$ leads to hyperventilation, drowsiness, and eventually death. ${ }^{83}$ These safety and environmental concerns solidify the need to monitor $\mathrm{CO}_{2}$ and $\mathrm{SO}_{2}$ concentrations.

\section{Box 3: $\quad \mathrm{CO}_{2} / \mathrm{SO}_{2}$ from volcanic}

\section{eruptions}

The 1783-1784 Laki (Skaftár Fires) volcanic eruption in Iceland was estimated to have released around $122 \mathrm{Mt}$ of $\mathrm{SO}_{2}$ and $350 \mathrm{Mt}$ of $\mathrm{CO}_{2}$, making it one of the largest natural atmospheric pollution events in history. ${ }^{84,85}$ Much of the crops and livestock in Iceland were affected by the eruption, ${ }^{85}$ leading to a famine and the death of ca. $20 \%$ of the human population on Iceland. ${ }^{85}$ The volcanic pollution spread over a large por- 


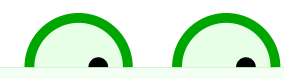

tion of the northern hemisphere, with evidence that both Alaska and China observed the sulfur aerosol plumes. ${ }^{85}$ As the ratio of $\mathrm{CO}_{2}$ to $\mathrm{SO}_{2}$ concentration in volcanic plume gas is predictive of the imminence of volcanic eruptions (as demonstrated in the Mount Etna and Stromboli volcanoes ${ }^{86,87}$ ), $\mathrm{CO}_{2} / \mathrm{SO}_{2}$ sensors could give people ample time to get to safety.

Fig. 1 is a cartoon of our sensor array comprised of $n=2$ QCMs monitoring the mass of adsorbed gas in their respective thin-film coats of MOFs. The sensor array is immersed in a $\mathrm{CO}_{2} / \mathrm{SO}_{2}$ mixture $(g=2)$ at room temperature $^{4}$. Our objective is to choose the optimal subset of MOFs to coat $n \in\{2,3\}$ QCMs to compose a robust sensor array that accurately predicts the partial pressures of $\mathrm{CO}_{2}$ and $\mathrm{SO}_{2}$ in the gas phase in spite of three challenges: (a) the concentrations of both $\mathrm{CO}_{2}$ and $\mathrm{SO}_{2}$ are variable, (b) all candidate MOFs for the sensor array adsorb both $\mathrm{CO}_{2}$ and $\mathrm{SO}_{2}$ appreciably (we must resort to a cross-sensitive sensor array), and (c) the $\mathrm{QCM}$ measurements and our estimates of Henry coefficients of $\mathrm{CO}_{2}$ and $\mathrm{SO}_{2}$ in the MOFs are corrupted by error. Fig. 3a displays the crystal structures of the set of $12 \mathrm{MOF}$ candidates we consider for coating the QCMs, chosen based on the availability of experimental $\mathrm{CO}_{2}$ and $\mathrm{SO}_{2}$ adsorption data near room temperature $(293 \mathrm{~K}$ to $298 \mathrm{~K}) .{ }^{25,90,96,99,101-105}$ To characterize the Henry coefficient matrix $\mathbf{H}$ in eqn. 2 for different selections of MOFs, we extracted $\mathrm{CO}_{2}$ and $\mathrm{SO}_{2}$ adsorption isotherms from plots in the literature, then identified their Henry coefficients by fitting a line with zero intercept to the low pressure data (see Sec. S1). Fig. $3 \mathrm{~b}$ shows the spread and correlation of the Henry coefficients of $\mathrm{CO}_{2}$ and $\mathrm{SO}_{2}, \mathrm{H}_{\mathrm{CO}_{2}}$ and $H_{\mathrm{SO}_{2}}$, respectively, among the MOF candidates. Most of the MOFs display a larger $\mathrm{H}_{\mathrm{SO}_{2}}$ than $\mathrm{H}_{\mathrm{CO}_{2}}$, with the exception of KAUST-7 and KAUST-8. KAUST-7 has the largest $\mathrm{H}_{\mathrm{CO}_{2}}$

\footnotetext{
${ }^{4}$ Equivalently, we assume the sensor is operating in air, but all constituents except $\mathrm{CO}_{2}$ and $\mathrm{SO}_{2}$ do not adsorb in the MOFs, making gas composition space $g=$ 2-dimensional.
}

(11.5 g/(g-bar)) whereas NOTT-300 has the largest $H_{\mathrm{SO}_{2}}(31.0 \mathrm{~g} /(\mathrm{g}$-bar $))$. Without loss of generality, we assume below that the same mass of MOF is deposited on each QCM.

\subsection{Curating an $n=2$ sensor ar- ray}

First, we determine the optimal pair of MOFs from Fig. 3a to coat $n=2$ QCMs. Excluding duplication of a MOF, there are 66 distinct pairs of MOFs to evaluate.

We visualize the map from composition space to sensor response space in Fig. 4 for an example pair of MOFs, \{MFM-300(In), Mg-MOF-74\}. The concentric circles in composition space are mapped to the concentric ellipses in response space, and a point with a given color in composition space is mapped to the same color in response space (inspired by domain coloring ${ }^{106}$ to visualize complex functions, see Sec. S3). This pair of MOFs gives rise to a cross-sensitive sensor array as in the caricature in Fig. 2(e). The right singular vectors $\mathbf{v}_{\mathbf{1}}$ and $\mathbf{v}_{\mathbf{2}}$ of $\mathbf{H}$ for this MOF pair show which directions in composition space elicit the largest and smallest responses, which are in the direction of $\mathbf{u}_{1}$ and $\mathbf{u}_{2}$, respectively. The largest sensor array response corresponds to an increase or decrease in both $p_{\mathrm{CO}_{2}}$ and $p_{\mathrm{SO}_{2}}$, whereas the smallest response occurs when $p_{\mathrm{CO}_{2}}$ increases and $p_{\mathrm{SO}_{2}}$ decreases or vice versa.

Comparing the linear maps $\mathbf{p}^{*} \mapsto \mathbf{m}^{*}$ under two different pairs of MOFs allows us to juxtapose their suitability for a sensor array. Fig. 5a shows a circle in composition space and its image under $\{$ NOTT-202a, KAUST-7 $\left(\sigma_{1}=11.9, \sigma_{2}=9.5\right)$ and MFM-600, MgMOF-74 $\left(\sigma_{1}=13.1, \sigma_{2}=1.4\right)$. The Henry coefficient matrix $\mathbf{H}$ of the latter pair harbors a smaller $\sigma_{2}$. Included in Fig. 5a is a hypothetical "error zone", representing the limit of detection of the QCM mass measurements; responses that fall in this error zone are indistinguishable from $\mathbf{m}^{*}=\mathbf{0}$ owing to measurement error/noise. The QCM-sensor based on (MFM$600, \mathrm{Mg}-\mathrm{MOF}-74\}\left(\sigma_{2}=1.4\right)$ maps the circle to an ellipse with a semi-minor axis that fails to escape the error zone; with this MOF pair, 


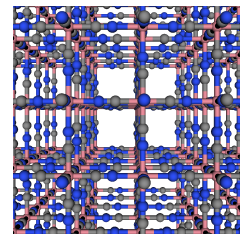

$\mathrm{Co}_{3}\left[\mathrm{Co}(\mathrm{CN})_{6}\right]_{2}{ }^{88}$

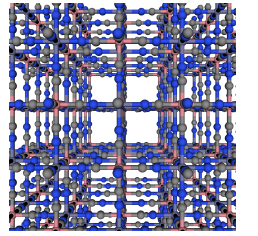

$\mathrm{Zn}_{3}\left[\mathrm{Co}(\mathrm{CN})_{6}\right]_{2}{ }^{94}$

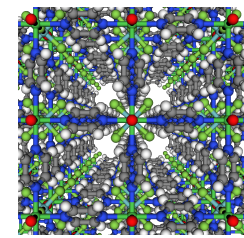

KAUST- $7^{89}$

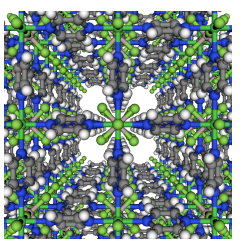

KAUST $-8^{95}$

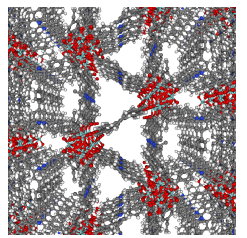

MFM- $600^{90}$

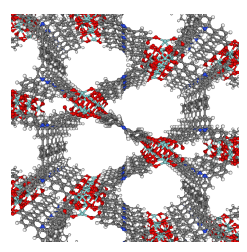

MFM- $601^{90}$

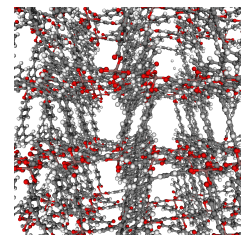

NOTT-202a ${ }^{91}$

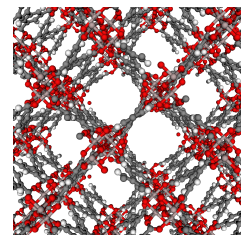

NOTT-300 ${ }^{96}$
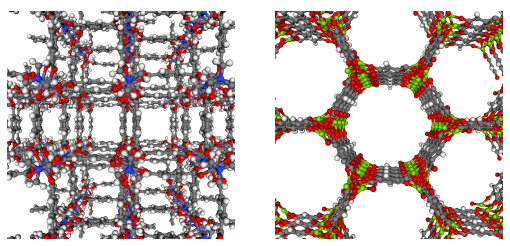

$\mathrm{Zn}(\mathrm{bdc})(\mathrm{ted}){ }_{0.5}{ }^{92} \mathrm{Mg}-\mathrm{MOF}-74^{93}$

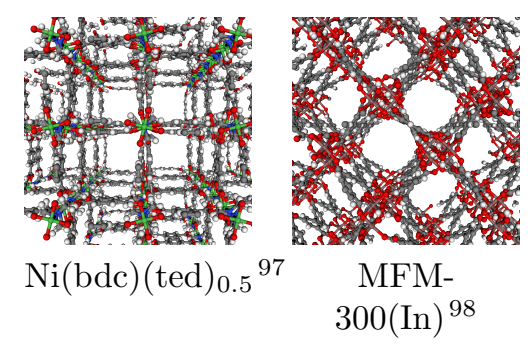

(a)

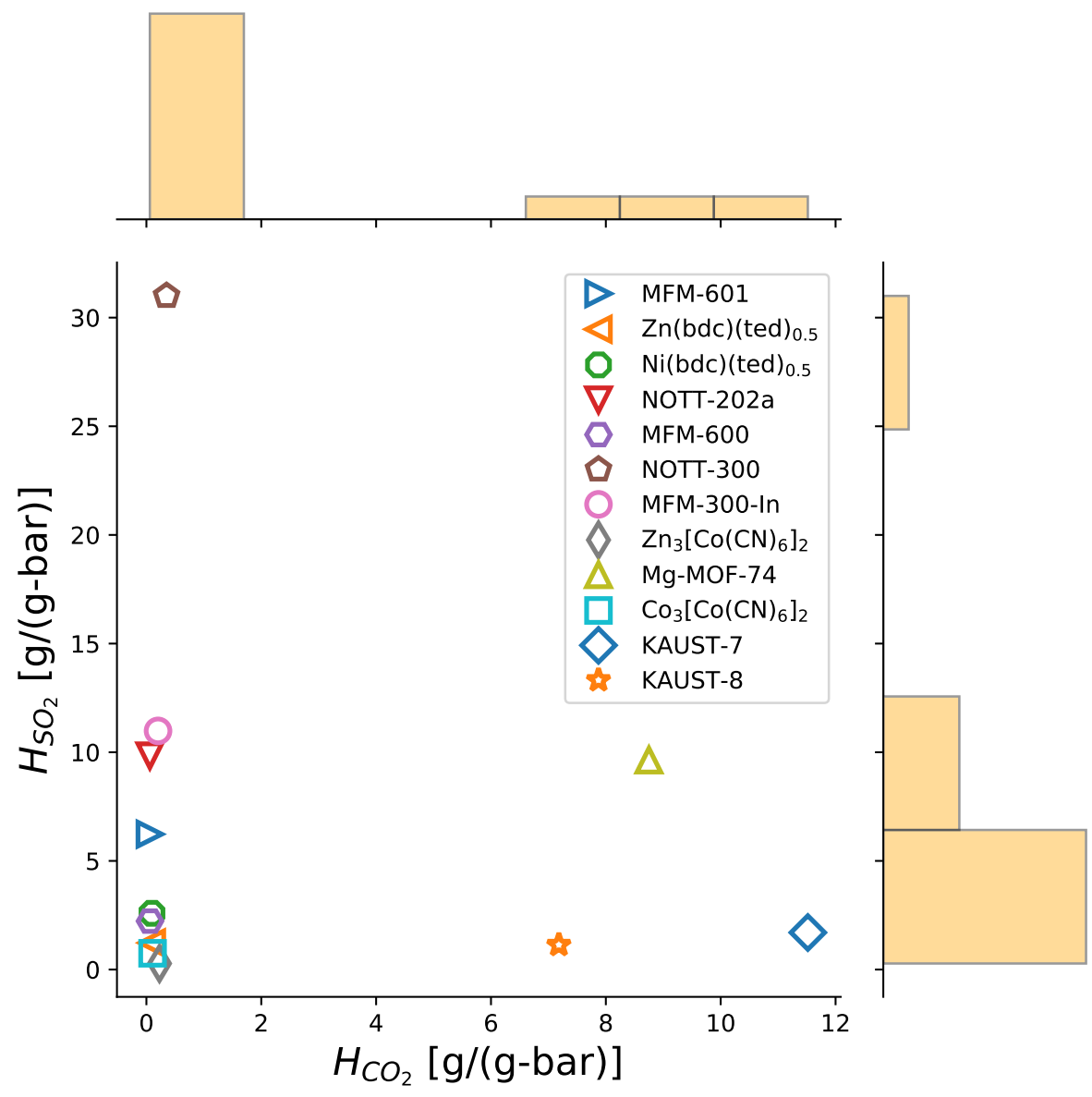

(b)

Figure 3: To demonstrate our methodology, we explore how to choose the optimal subset of two/three MOFs to compose a robust QCM-MOF sensor array for determining the concentrations of $\mathrm{CO}_{2}$ and $\mathrm{SO}_{2}$ in the gas phase. Twelve MOFs, ${ }^{89-93,95,97-101}$ whose crystal structures are shown in (a), are candidates as recognition elements in the sensor array of QCM-MOFs. The Henry coefficients of $\mathrm{CO}_{2}$ and $\mathrm{SO}_{2}$ in the MOFs, whose correlation (scatter plot) and spread (marginal histograms [orange]) are in (b), will inform the selection of the MOFs for the sensor array. These Henry coefficients were extracted from experimentally reported adsorption isotherms near room temperature. ${ }^{25,90,96,99,101-105}$ 

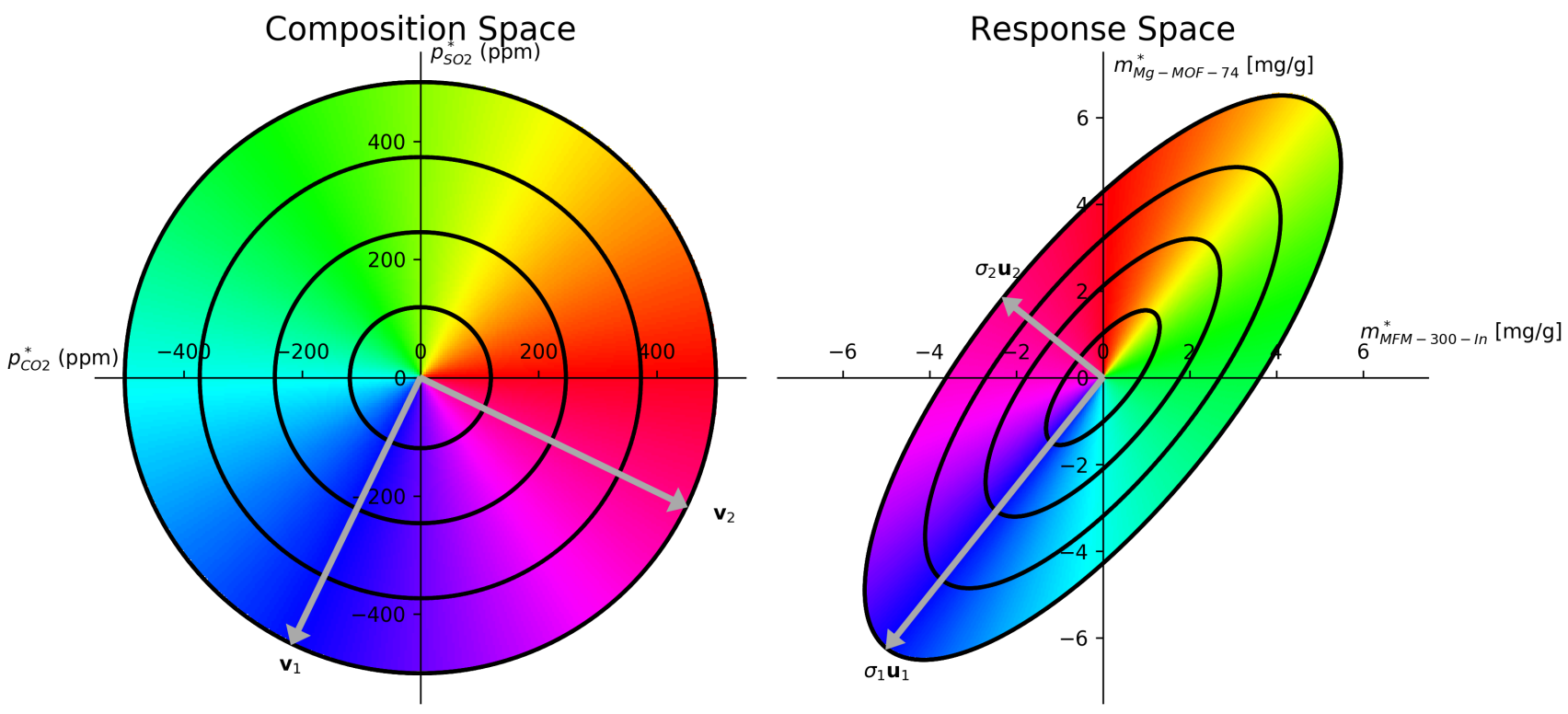

Figure 4: The map from composition space (left) to sensor array response space (right) via the Henry coefficient matrix $\mathbf{H}$ of a QCM-MOF sensor array in Fig. 1 using Mg-MOF-74 and MFM300(In). A point of a given color in composition space is mapped to the same color in sensor array response space; also, the concentric circles on the left are mapped to the concentric ellipses on the right. The right singular vectors of $\mathbf{H}, \mathbf{v}_{1}$ and $\mathbf{v}_{2}$, are mapped to the responses $\sigma_{1} \mathbf{u}_{1}$ and $\sigma_{2} \mathbf{u}_{2}$, respectively, which are its corresponding left singular vectors scaled by their cognate singular values. Note the anisotropy in the sensor array response; changes in composition space of a given magnitude in the direction of $\mathbf{v}_{1}$ and $\mathbf{v}_{2}$ elicit the largest and smallest, respectively, sensor array responses $\left(\sigma_{1}=16.0, \sigma_{2}=5.9\right)$.

some composition changes on the circle fail to generate a response discernible from $\mathbf{m}^{*}=\mathbf{0}$. Meanwhile, the QCM-sensor based on NOTT202a, KAUST-7 $\}\left(\sigma_{2}=9.6\right)$ maps the circle to an ellipse with a longer semi-minor axis that escapes the error zone. Therefore, this MOF pair results in a more robust sensor, as it elicits a response that is distinguishable from $\mathbf{m}^{*}=\mathbf{0}$ regardless of the direction of the change in composition space.

Comparing the reverse linear maps $\mathbf{m}^{*} \mapsto \mathbf{p}^{*}$ (straightforward only for determined systems where $\mathbf{H}^{-1}$ exists) under two pairs of MOFs is also an instructive juxtaposition of their suitability for a sensor array. For the same MOF pairs appearing in Fig. 5a, Fig. 5b shows the mapping of the boundary of the "error zone" back into composition space by $\mathbf{H}^{-1}$; the resulting ellipses encompass all points in compo- sition space whose responses we cannot distinguish from $\mathbf{m}^{*}=\mathbf{0}$. The QCM-sensor based on \{MFM-600, Mg-MOF-74\}, whose $\mathbf{H}$ possesses the smallest small singular value $\left(\sigma_{2}=1.4\right)$, has a much larger region in composition space that cannot be distinguished from $\mathbf{m}^{*}=\mathbf{0}$ compared to a QCM-sensor based on $\{$ NOTT-202a, KAUST-7\}.

Fig. 5 reinforces that the singular values of $\mathbf{H}$ are essential for comparing two QCM-MOF sensor arrays. Considering the operator norm of $\mathbf{H}$ in eqn. 9, in Fig. 5a we desire a pair of MOFs that harbors a large $\sigma_{2}$ so the responses to all compositions will escape the "error zone". Under the reverse mapping in Fig. 5b, since $\left\|\mathbf{H}^{-1}\right\|_{o p}=1 / \sigma_{2}(\mathbf{H})$, the MOF pair whose $\sigma_{2}(\mathbf{H})$ is smallest will most amplify the error zone, leading to more drastic composition changes whose responses cannot be dis- 
tinguished from $\mathbf{m}^{*}=\mathbf{0}$.

Finally, in Fig. 6, we conduct a visual highthroughput screening of MOF pairs for a sensor array by, for all 66 possible pairs, constructing $\mathbf{H}$, then (i) as in Fig. 4, visualizing the mapping of a circle in composition space to an ellipse in sensor array response space and (ii) computing $\sigma_{2}(\mathbf{H})$ arising from that pair. The length of the semi-minor axis of each ellipse in response space is determined by $\sigma_{2}(\mathbf{H})$; the blue bar above each ellipse represents the relative magnitude of $\sigma_{2}(\mathbf{H})$ among the pairs. The optimal pair of MOFs to compose a robust sensor array is \{KAUST-7, NOTT-300\}, highlighted yellow in Fig. 4, because its $\sigma_{2}(\mathbf{H})$ is the largest of all pairs. Sensor arrays containing MOFs such as $\mathrm{Zn}\left[\mathrm{Co}(\mathrm{CN})_{6}\right]_{2}, \mathrm{Co}\left[\mathrm{Co}(\mathrm{CN})_{6}\right]_{2}, \mathrm{Zn}(\mathrm{bdc})(\text { ted })_{0.5}$, MFM-600, and $\mathrm{Ni}(\mathrm{bdc})(\text { ted })_{0.5}$ are comparatively poor because of the small Henry coefficients of both $\mathrm{CO}_{2}$ and $\mathrm{SO}_{2}$ in these MOFs (see Fig. 3b) that render their responses insensitive to changes in gas composition. Each MOF in the pairs that tend to form the most robust sensor arrays (Mg-MOF-74, KAUST-7, KAUST-8, NOTT-300, MFM-300-In, NOTT-202a, MFM601) tends to exhibit larger Henry coefficients of both gases, rendering their responses sensitive to changes in gas composition in all directions. Notably, the mapping of a circle under $\{$ NOTT-300, KAUST-7 $\}$ is an ellipse whose semi-axes are nearly aligned with the coordinate axes, resembling the ideal sensor array in Fig. 2(d). Indeed, NOTT-300 has a high affinity for $\mathrm{SO}_{2}$ and a low affinity for $\mathrm{CO}_{2}$, while KAUST-7 has a high affinity for $\mathrm{CO}_{2}$ and a low affinity for $\mathrm{SO}_{2}$. On the other hand, relatively robust sensor arrays based on MOF pairs \{Mg-MOF-74, KAUST-7\}, \{NOTT-300, MgMOF-74\}, and $\{$ NOTT-202a, Mg-MOF-74 $\}$ are highly cross-sensitive and possess ellipses whose semi-axes are not aligned with the coordinate axes; indeed, Fig. 3b shows that the Henry coefficients of both $\mathrm{CO}_{2}$ and $\mathrm{SO}_{2}$ in Mg-MOF-74 are large. Still, though both $\mathrm{CO}_{2}$ and $\mathrm{SO}_{2}$ contribute to the response of these cross-sensitive QCM-MOF sensors, we can accurately determine the contribution by each and thus the gas composition $\mathbf{p}$ by solving $\mathbf{m}=\mathbf{H} \mathbf{p}$.

\subsubsection{Response anisotropy and non-} robust sensors

We focused on identifying pairs of MOFs that form robust sensor arrays, i.e., that elicit a response of large magnitude regardless of the direction moved in composition space, hence the focus on $\sigma_{2}(\mathbf{H})$ (see eqn. 10). We remark that, if we aim to detect only a certain composition change- i.e., only when $\mathbf{p}^{*}$ points in a certain direction- we would instead screen pairs of MOFs based on $\left\|\mathbf{H} \mathbf{p}^{*}\right\|$ for that particular direction, as in Ref. 65. Such a scenario might arise when the source/sink of $\mathrm{CO}_{2}$ and $\mathrm{SO}_{2}$ is expected from a set of well-characterized reactions that constrain the stoichiometry. As the sensor array response can be highly anisotropic, then, the most robust sensor array does not necessarily elicit a larger response in the typical direction $\mathbf{p}^{*}$ (e.g., compare the linear maps of $\{$ Mg-MOF-74, KAUST-7 $\}$ to $\{$ MFM-300-In, KAUST-8 $\}$ in Fig. 6).

\subsection{Curating an $n=3$ sensor ar- ray}

We now determine the optimal triad of MOFs from Fig. 3a to coat $n=3$ QCMs. There are 220 distinct triads, excluding the duplication of a MOF. Owing to inevitable measurement and model error, such an array composed of more sensors than gases $(n=3>2=g)$ gives rise to an overdetermined system, necessitating least squares to predict the gas composition from the array response (see Sec. 2.2.3). Still, however, in Sec. 2.4.2, we argued that the MOF triad with the largest $\sigma_{2}(\mathbf{H})$ yields the optimal sensor array.

Fig. 7 displays the mapping of a circle in composition space by the Henry coefficient matrix $\mathbf{H}$ of a sensor array using $\{\mathrm{MFM}-300(\mathrm{In}), \mathrm{Mg}-$ MOF-74, KAUST-8\}. As in Fig. 2(f), the image is an ellipse on a plane (orange, column space of $\mathbf{H}$ ) embedded in the 3D response space. Owing to measurement and model error, the measured response $\mathbf{m}^{*}$ is unlikely to lie on this plane, hence the necessity of least squares optimization to predict the gas composition; eqn. 6 projects the response $\mathbf{m}^{*}$ onto this plane to 


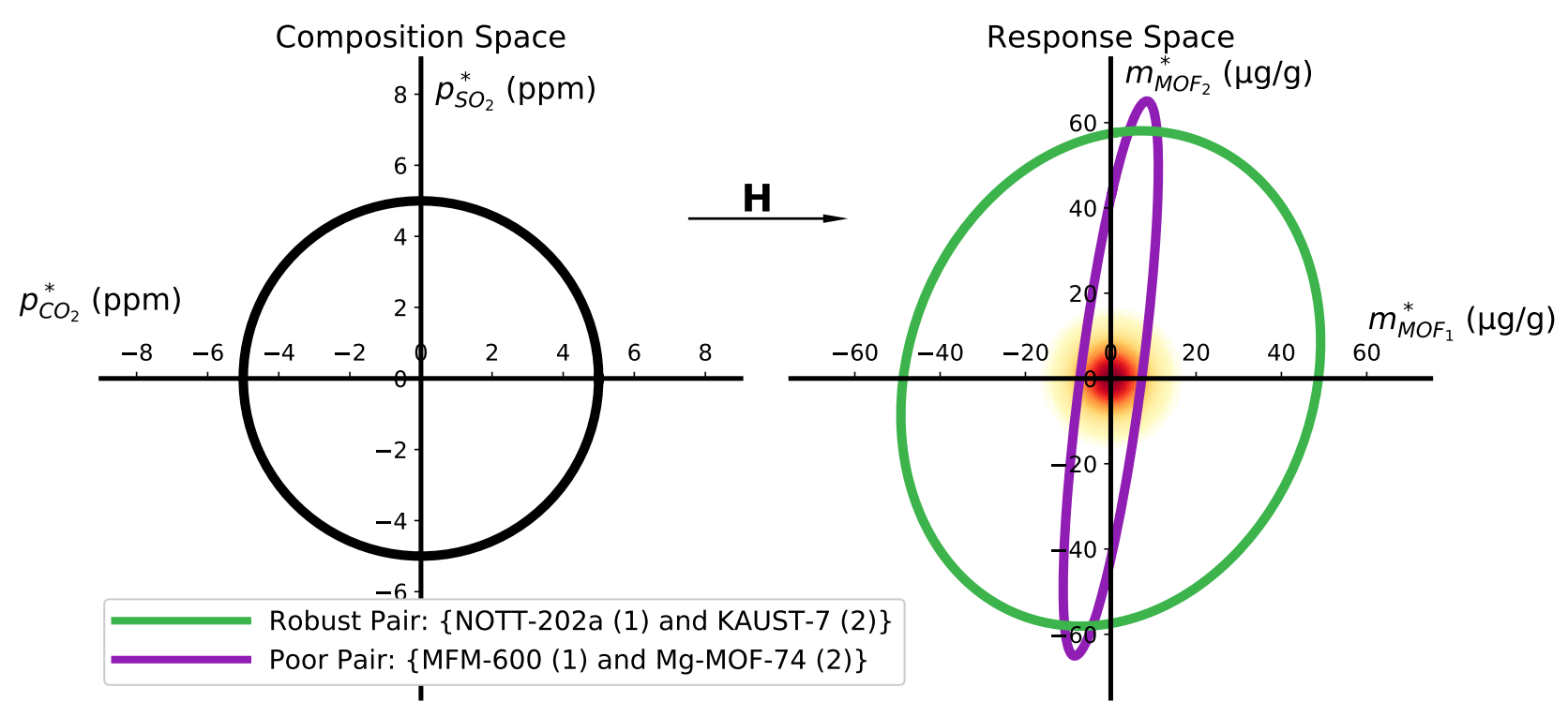

(a)

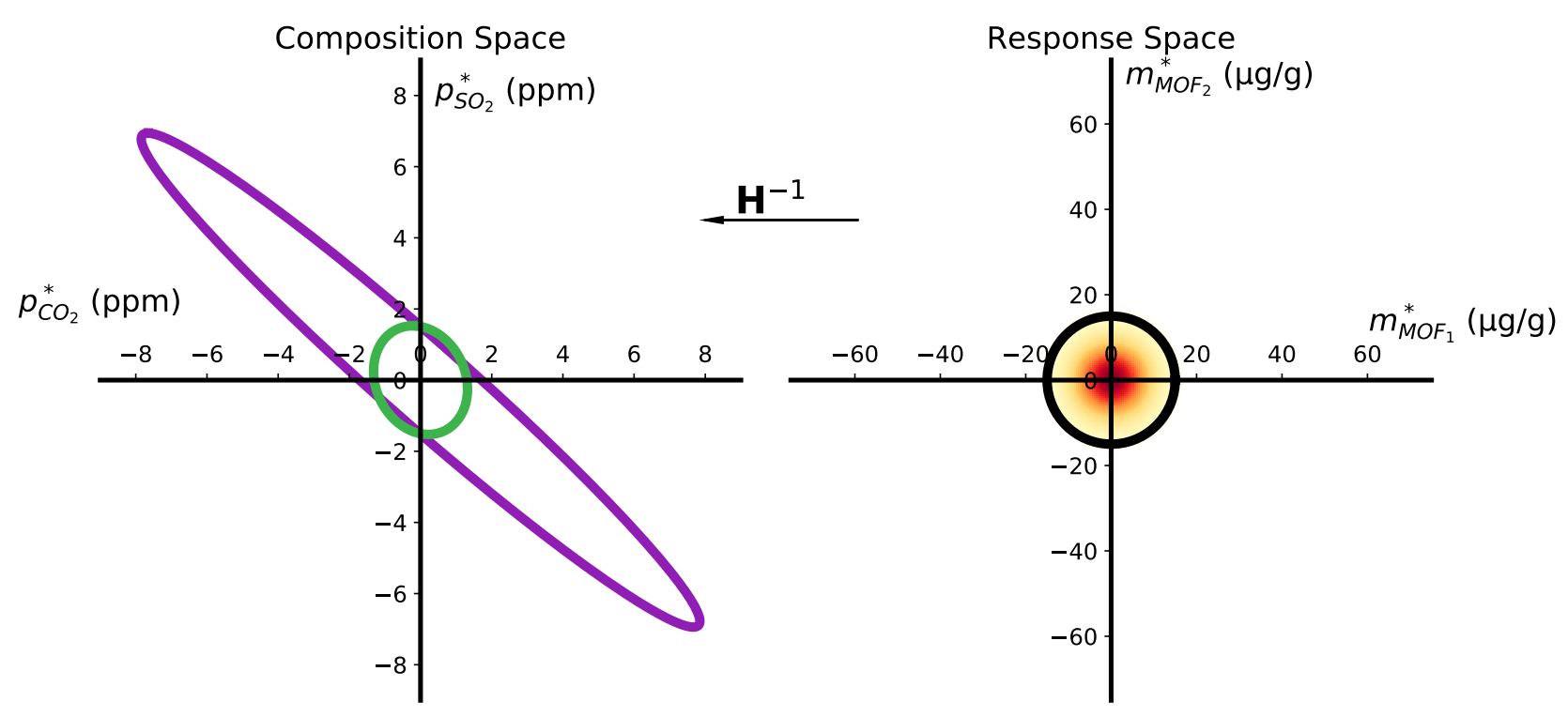

(b)

Figure 5: Juxtaposing two MOF pairs [green: $\{$ NOTT-202a, KAUST-7 $\}\left(\sigma_{1}=11.9, \sigma_{2}=9.5\right)$, purple: $\left\{\right.$ MFM-600, Mg-MOF-74\} $\left.\left(\sigma_{1}=13.1, \sigma_{2}=1.4\right)\right]$ via their [invertible here] linear map between gas composition space (left) and sensor array response space (right) dictated by their respective Henry coefficient matrix $\mathbf{H}$. The orange heatmap represents an error zone; owing to measurement noise/error, we cannot confidently distinguish a response in the error zone from $\mathbf{m}^{*}=\mathbf{0}$. (a) The circle in composition space (black) is mapped to response space by the two MOF pairs (green, purple). The response of the more robust pair (green) escapes the error zone, whereas the response of the poorly robust pair (purple) fails to escape the error zone for some composition changes. (b) The boundary of the error zone (black) is mapped to composition space for the two MOF pairs (green, purple) via their respective $\mathbf{H}^{-1}$. The image of the boundary of the error zone in response space into composition space is larger for the poorly robust pair (purple), indicating there is a larger region in composition space that elicits a response indistinguishable from $\mathbf{m}^{*}=\mathbf{0}$. 


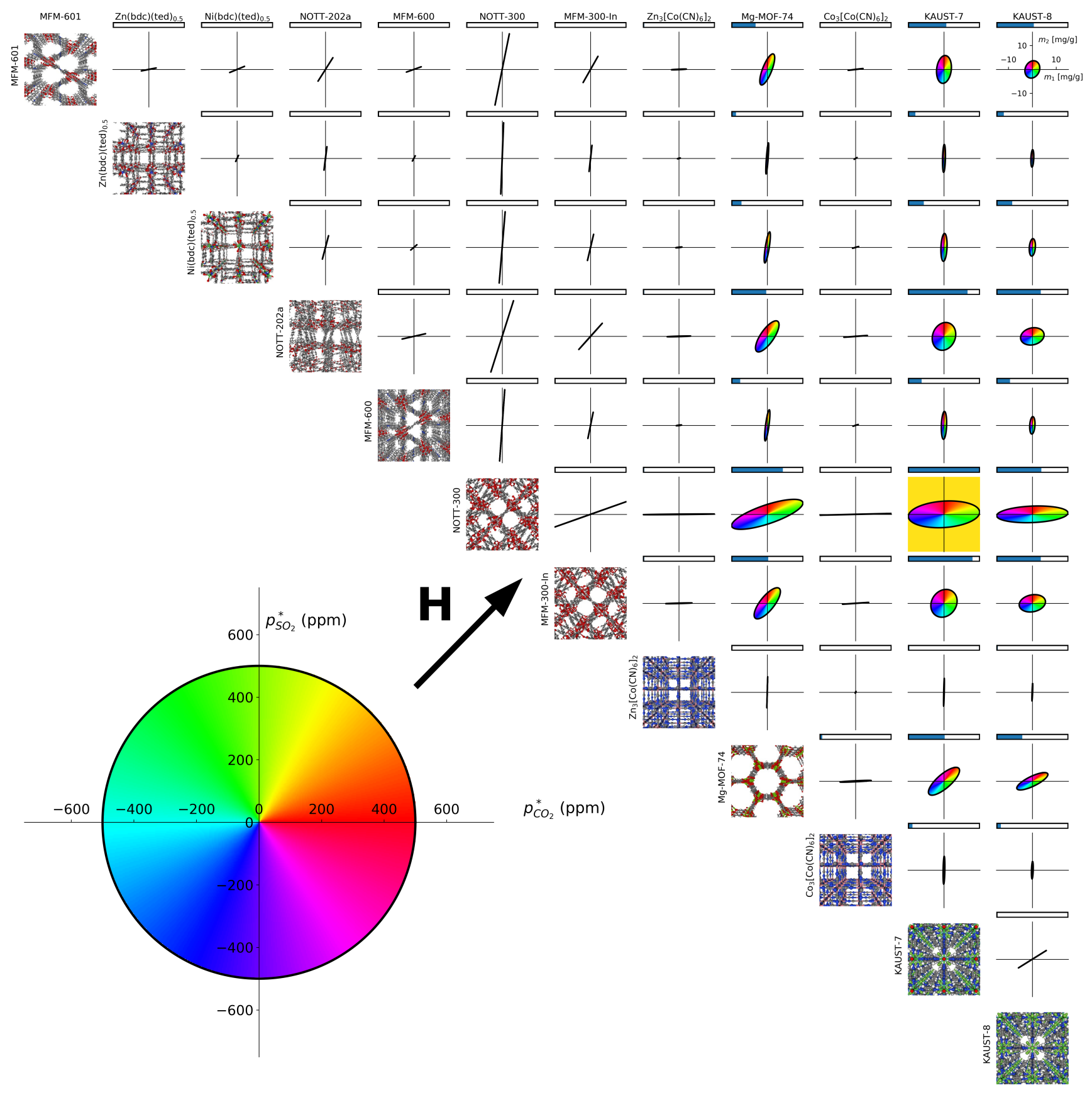

Figure 6: High-throughput screening of MOF pairs for a sensor array for $\mathrm{CO}_{2}$ and $\mathrm{SO}_{2}$ by visualizing the linear map of a circle in gas composition (bottom left) to an ellipse an sensor response space (panels in upper diagonal). A point of a given color in composition space is mapped to the same color in sensor response space. The pair whose response is shown is determined by the row and column, with MOF crystal structures on the diagonal. The top right panel displays the scale shared among all response space axes. The bars above each ellipse are colored blue proportionally to the smallest singular value $\sigma_{2}$ of $\mathbf{H}$ for that pair. Because its $\sigma_{2}(\mathbf{H})$ is the largest, \{KAUST-7, NOTT-300\}, highlighted yellow, forms the most robust sensor array.

arrive at a prediction of the composition, $\mathbf{p}^{+}$. Through eqn. 10, $\sigma_{2}(\mathbf{H})$ determines the length of the semi-minor axis of the response ellipse in Fig. 7.
As the most robust $n=3$ sensor array elicits a response of large magnitude for all directions moved in composition space of a given magnitude, from a geometric perspective, the most ro- 

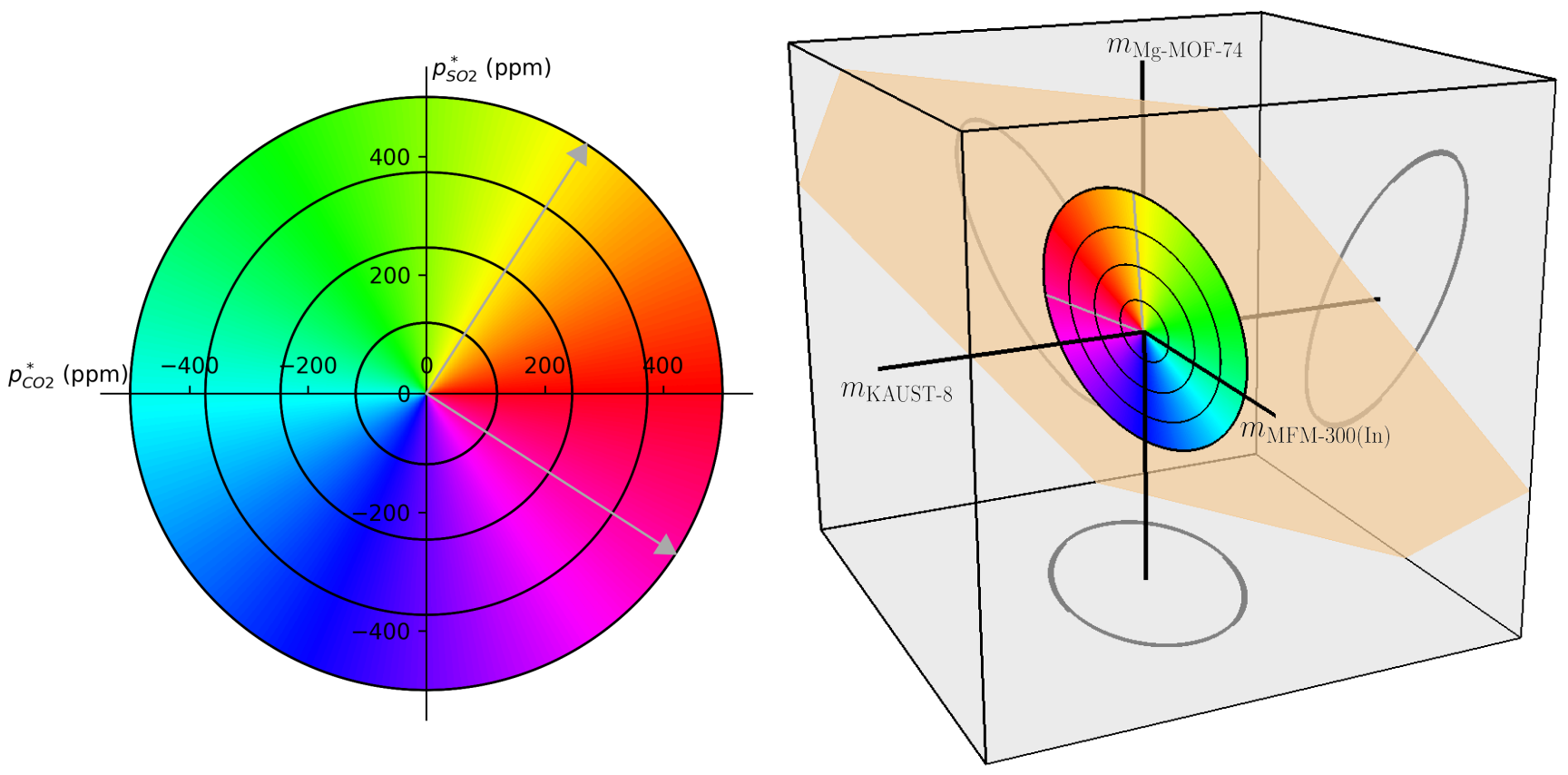

Figure 7: A visualization of the mapping from composition space (left) to sensor array response space (right) dictated by a tall $(n=3 \times 2=g)$ Henry coefficient matrix $\mathbf{H}$ using $\{$ MFM-300(In), Mg-MOF-74 and KAUST-8\}. A point of a given color in composition space is mapped to the same color in sensor response space. A circle in 2D gas composition space is mapped to a $2 \mathrm{D}$ ellipse embedded in 3D sensor array response space. The $2 \mathrm{D}$ ellipse is projected onto the walls of the bounding box. The orange surface shows the plane on which the ellipse lies and represents the column space of $\mathbf{H}$.

bust triad of MOFs will give rise to the largest $\sigma_{2}(\mathbf{H})$. Exhaustively exploring all 220 possible MOF triads for a sensor array by constructing the $\mathbf{H}$ for that triad and computing $\sigma_{2}(\mathbf{H})$, we find \{NOTT-300, KAUST-7, Mg-MOF-74\} forms the optimal triad of MOFs; not surprisingly, the most robust triad includes the optimal pair of MOFs to yield a robust $n=2$ QCM-MOF sensor in Fig. 6.

\subsection{Curating an $n>3$ sensor ar- ray}

While it is difficult to visualize the linear map $\mathbf{p}^{*} \mapsto \mathbf{m}^{*}$ in dimensions greater than three, our methodology to curate MOFs for robust sensor arrays holds for $n, g>3$ as well; select the subset of MOFs yielding the largest $\sigma_{g}(\mathbf{H})$ (take $n \geq g$ ).

\subsection{Can an additional sensor be detrimental?}

An important question is whether adding an additional sensor to an incumbent sensor array can be a detriment to its prediction of the gas composition.

First, we show that adding another QCM coated with a MOF with a nonzero Henry coefficient increases the smallest singular value of the Henry coefficient matrix of the sensor array. Consequently, the bounds on the norm of the error in the predicted gas composition $\|\delta \mathbf{p}\|$ for a given $\|\delta \mathbf{m}\|,\|\delta \mathbf{H}\|_{o p}$, and $\|\mathbf{e}\|$, found in eqns. 14 and 17, can only be tightened by an additional sensor. Further, from the linear map perspective in Fig. 5, the minimum-norm sensor array response will be more likely to escape the error zone when an additional sensor is added. Let $\mathbf{H}_{0}$ be the $n-1 \times g$ Henry coefficient of an incumbent sensor array comprised of $n-1$ QCM-MOFs and exposed to a gas mixture with 
$g$ species. Upon adding another QCM-MOF sensor to the array, the Henry coefficient matrix becomes:

$$
\mathbf{H}=\left[\begin{array}{l}
\mathbf{H}_{0} \\
\mathbf{H}_{a}
\end{array}\right]
$$

where $\mathbf{H}_{a}$ is the $1 \times g$ row contributed by the additional sensor. To relate the smallest singular value of $\mathbf{H}$ to that of $\mathbf{H}_{0}$, note:

$$
\|\mathbf{H} \mathbf{p}\|^{2}=\left\|\mathbf{H}_{0} \mathbf{p}\right\|^{2}+\left\|\mathbf{H}_{a} \mathbf{p}\right\|^{2} .
$$

for all $\mathbf{p} \in \mathbb{R}^{g}$. Dividing by $\|\mathbf{p}\|^{2}$ where $\mathbf{p} \neq \mathbf{0}$, taking the minimum of both sides, and noting the minimizer could differ for the two terms on the right:

$$
\min _{\mathbf{p} \neq \mathbf{0}} \frac{\|\mathbf{H} \mathbf{p}\|^{2}}{\|\mathbf{p}\|^{2}} \geq \min _{\mathbf{p} \neq \mathbf{0}} \frac{\left\|\mathbf{H}_{0} \mathbf{p}\right\|^{2}}{\|\mathbf{p}\|^{2}}+\min _{\mathbf{p} \neq \mathbf{0}} \frac{\left\|\mathbf{H}_{a} \mathbf{p}\right\|^{2}}{\|\mathbf{p}\|^{2}}
$$

Recognizing that the minimum of the square of a function with a positive range is the square of that function's minimum, we arrive at a relationship between the smallest singular value of $\mathbf{H}$ and that of its two submatrices $\mathbf{H}_{0}$ and $\mathbf{H}_{a}$ via eqn. 10:

$$
\left[\sigma_{g}(\mathbf{H})\right]^{2} \geq\left[\sigma_{g}\left(\mathbf{H}_{0}\right)\right]^{2}+\left[\sigma_{1}\left(\mathbf{H}_{a}\right)\right]^{2},
$$

where $n-1 \geq g$ is assumed. We conclude that adding another QCM coated with a MOF exhibiting a nonzero Henry coefficient (guaranteeing $\sigma_{1}\left(\mathbf{H}_{a}\right) \neq 0$ ) to a given sensor array serves to increase the smallest singular value of its Henry coefficient matrix.

Second, we explicitly model the mass measurement errors by the QCMs as independent and normally distributed random variables with variance $\gamma^{2}$ and show that an additional sensor in the array leads to, on average, a more accurate prediction of the gas composition. Let $\mathbf{m}$ denote the true masses of gas adsorbed in the MOFs and $\mathbf{p}$ denote the true gas composition, so that $\mathbf{m}=\mathbf{H p}$ (neglecting errors in $\mathbf{H})$. Take the measured sensor array response as $\mathbf{m}+\delta \mathbf{m}$, with $\delta \mathbf{m} \sim \mathcal{N}\left(\mathbf{0}, \gamma^{2} \mathbf{I}\right)$ the measurement error. As a result, the predicted gas composition differs from the truth $\mathbf{p}$ by random vector $\delta$ p. Eqn. 15 therefore holds (taking $\delta \mathbf{H}=\mathbf{0}$ and despite subtle differences in the definitions of the variables), and we find $\delta \mathbf{p}=\mathbf{H}^{+} \delta \mathbf{m}$. We aim to find how the expected norm of $\delta \mathbf{p}$ under the measurement error $\delta \mathbf{m}$ depends on the properties of $\mathbf{H}$ :

$$
\begin{aligned}
\left\langle\|\delta \mathbf{p}\|^{2}\right\rangle & =\left\langle(\delta \mathbf{m})^{\top}\left(\mathbf{H}^{+}\right)^{\top} \mathbf{H}^{+} \delta \mathbf{m}\right\rangle \\
& =\gamma^{2} \operatorname{Tr}\left[\left(\mathbf{H}^{+}\right)^{\top} \mathbf{H}^{+}\right] \\
& =\gamma^{2}\left\|\mathbf{H}^{+}\right\|_{\mathrm{F}}^{2} \\
& =\gamma^{2} \sum_{i=1}^{g} \frac{1}{\left[\sigma_{i}(\mathbf{H})\right]^{2}}
\end{aligned}
$$

where $\operatorname{Tr}(\cdot)$ is the trace, $\|\cdot\|_{\mathrm{F}}$ is the Frobenius norm, and $n \geq g$ is assumed. Again, the singular values of $\mathbf{H}$ play a central role in determining the expected norm of the error in the predicted gas composition under mass measurement error. In Sec. S5, we use a relation between the eigenvalues of a symmetric matrix and a rank-one perturbation of it ${ }^{107}$ to show that, in the context of eqn. 19, $\sigma_{i}(\mathbf{H}) \geq \sigma_{i}\left(\mathbf{H}_{0}\right)$ for $i=1, \ldots, g$. Eqn. 26 therefore implies that $\left\langle\|\delta \mathbf{p}\|^{2}\right\rangle$ decreases when an additional sensor is added to an incumbent array. This analysis neglects model error that could emanate from adding a MOF to the array where $\mathbf{p}$ is outside of its Henry regime.

\section{Extension beyond the Henry regime}

Until now, we applied Henry's law under the assumption of dilute conditions. While Henry's law gave rise to a linear model (eqn. 2) convenient for analysis, in practice, equilibrium adsorption in MOFs is nonlinear and saturates as pores are filled with gas. We show that our modeling framework can be adapted to address these deficiencies by linearizing a nonlinear adsorption model that accounts for saturation. Such a linearized model is valid only near the nominal baseline gas composition $\overline{\mathbf{p}}$ about which we perform the Taylor expansion.

For example, a non-linear model for equilibrium multicomponent adsorption of $g$ gases in a MOF offering $M$ identical, independent adsorption sites is the competitive Langmuir ad- 
sorption model: ${ }^{108}$

$$
m(\mathbf{p})=M \frac{\mathbf{K}^{\top} \mathbf{p}}{1+\mathbf{K}^{\top} \mathbf{p}},
$$

where $m$ is the total mass of gas adsorbed and $\mathbf{K}$ is the vector of Langmuir constants describing the affinity of the $g$ gases for the adsorption site. In contrast to Henry's law in eqn. 1, eqn. 27 accounts for saturation as the adsorption sites fill with gas. Approximating eqn. 27 with a first-order Taylor polynomial around the baseline gas phase composition $\overline{\mathbf{p}}$ gives rise to the linearized adsorption model:

$$
m(\mathbf{p}) \approx m(\overline{\mathbf{p}})+\frac{1}{\left(1+\mathbf{K}^{\top} \overline{\mathbf{p}}\right)^{2}} M \mathbf{K}^{\top}(\mathbf{p}-\overline{\mathbf{p}}),
$$

which geometrically approximates $m(\mathbf{p})$ with a hyperplane passing through $m(\overline{\mathbf{p}})$ and tangent to $m(\mathbf{p})$. As $\overline{\mathbf{p}} \rightarrow \mathbf{0}$, we recover Henry's law $m(\mathbf{p})=M \mathbf{K}^{\top} \mathbf{p}$. As the adsorption sites saturate with gas, using eqns. 3 and $4,(1+$ $\left.\mathbf{K}^{\top} \overline{\mathbf{p}}\right)^{-2} M \mathbf{K}^{\top}$ behaves as a vector of pseudoHenry coefficients and diminishes as $\overline{\mathbf{p}}$ increases. Therefore, a MOF operating near the saturation regime would result in small pseudoHenry coefficients for all gases, and, from the perspective of Fig. 6, elimination of this candidate since the magnitude of its (insensitive) response to a change in the gas composition would be too small to distinguish from measurement error.

\section{Box 4: Fabricating QCM-MOF sen-} sors

Current synthesis methods for QCMMOF structures can be classified into two categories. The first is indirect synthesis, where prepared, powdery MOF crystals are physically loaded onto a QCM. ${ }^{29,109}$ However, the stability of a QCM-MOF structure prepared this way is questionable. The second is direct synthesis, where MOF thin films are chemically synthesized on a prepared surface. To encourage MOF growth on the QCM substrate, its $\mathrm{Au}$ surface is prepared either with a self-assembled monolayer (SAM) exposing appropriate func-

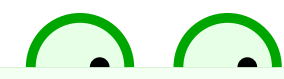

tional groups ${ }^{22,23,110-118}$ such as $\mathrm{COOH}$ or plating it with an appropriate metal such as $\mathrm{Cu}$ for growth of $\mathrm{Cu}_{3}(\mathrm{BTC})_{2} \cdot{ }^{21} \mathrm{SAMs}$ typically consist of long, thiol-based organic chains and attach to surfaces of gold. The growth of a thin film of MOF is then achieved through a solution-based reactioneither conventional, one-pot solvothermal synthesis $^{22,23}$ or step-by-step (cycling between metal and organic linker precursor solutions) liquid phase epitaxy ${ }^{110-118}$ on a SAM- or electrochemical deposition of the MOF on a pre-coated metal layer. ${ }^{21}$ Due to its relatively strong adhesion of the MOF layers with preferred growth orientation, SAMs are most widely adopted in MOF thin film fabrication. In consideration of sensor response time, it is crucial to determine an appropriate thickness of MOF films on QCMs. ${ }^{109}$ Fabrication of the thin film in a stepwise/additive manner, such as layer-bylayer liquid phase epitaxy, lends control over the film thickness during synthesis, and, in addition, is able to be automated.

\section{Discussion}

We mathematically framed a sensor array problem under dilute conditions: in the face of measurement error, aiming to accurately determine the composition of a gas phase with $g$ constituents from measurements of the (i) Henry coefficients of the $g$ gases in the $n$ MOFs (as calibration) and (ii) adsorbed masses in the $n$ MOFs by the QCMs (during deployment). We viewed $n$ QCM-MOF sensors as a linear map from $g$-dimensional gas composition space to $n$-dimensional sensor response space, which lent intuition for curating MOFs for sensor arrays. Given a large set of MOF candidates, we showed that the optimal subset of MOFs to coat QCMs to compose a sensor array form a Henry coefficient matrix $\mathbf{H}$ with the largest smallest singular value, guaranteeing (a) the most insensitivity of the predicted gas composition to measurement error and (b) a large response to changes in gas composition in all di- 
rections (so as to distinguish the response from mass measurement noise/error). We deployed our insights and demonstrated the curation of pairs/triads of MOFs to coat $n \in\{2,3\}$ QCMs for $\mathrm{CO}_{2} / \mathrm{SO}_{2}$ sensor arrays, given the MOF candidates in Fig. 3a. This culminated in a visual high-throughput screening in Fig. 6 that displayed the linear maps of circles in composition space to ellipses in response space for each candidate pair. The singular value decomposition of $\mathbf{H}$ was crucial for interpreting the linear map $\mathbf{p}^{*} \mapsto \mathbf{m}^{*}$, bounding errors in predicted gas composition resulting from perturbations in the measurements, evaluating the robustness of sensor arrays, and understanding the anisotropy of the response. Lastly, we outlined mathematical arguments to show that adding another sensor to an incumbent array results in, on average, a more accurate prediction of the gas composition, provided that (a) adsorption in the additional MOF obeys Henry's law and (b) the mass measurement error on the additional QCM is identical to the other QCMs in the array.

One may propose a simpler strategy to $\mathrm{cu}^{-}$ rate MOFs for a sensor array solely on the basis of their adsorptive selectivity: e.g., for a two-sensor array for $\mathrm{CO}_{2}$ and $\mathrm{SO}_{2}$, select the MOF with the highest $\mathrm{CO}_{2} / \mathrm{SO}_{2}$ selectivity and the MOF with the highest $\mathrm{SO}_{2} / \mathrm{CO}_{2}$ selectivity. This strategy fortuitously recovers from Fig. 3b KAUST-7, which has the highest selectivity for $\mathrm{CO}_{2}$, as a member of the optimal MOF pair from Sec. 3.1. However, the other member of the optimal MOF pair, NOTT-300, has only the third highest selectivity for $\mathrm{SO}_{2}$. We show two more examples in Fig. S12 (see Sec. S2) where choosing MOFs for a sensor array based on their adsorptive selectivities fails to recover the optimal pair. The magnitude of the Henry coefficients must be considered as well, as a large Henry coefficient equips a MOF with high sensitivity to changes in the gas composition. Thus, while not as simple as a curation heuristic based on adsorptive selectivity, the singular value decomposition of $\mathbf{H}$ is a reliable tool to identify the optimal subset of MOFs for a robust and accurate sensor array.

Our work has several limitations. Most im- portantly, while the adsorption model in eqn. 1 lends the instructive view of sensor arrays as a linear mapping, it is valid only at dilute conditions- a restrictive assumption. Henry's law does not account for saturation of the sensor response; when the pores of the MOF are nearly filled with gas, the mass of gas adsorbed in the MOF is insensitive to changes in the gas phase composition- detrimental for sensing. Our linearized model in Sec. 4 accounts for saturation by systematically assigning a small "pseudo-"Henry coefficient to a MOF whose pores are nearly full of gas molecules at the baseline composition $\overline{\mathbf{p}}$. However, such a linearized model is valid approximately only in a small neighborhood in composition space surrounding the baseline condition $\overline{\mathbf{p}}$. Mixed gas adsorption isotherms $m(\mathbf{p})$ could be highly non-linear and non-additive in contributions by each species due to e.g., cooperative adsorption, ${ }^{119,120}$ pore filling, ${ }^{121}$ or guestinduced linker rotation, ${ }^{122}$ or discontinuous due to e.g., guest-induced structural backbone transitions. ${ }^{123}$ Second, the Henry coefficients of $\mathrm{CO}_{2}$ and $\mathrm{SO}_{2}$ in the MOFs displayed in Fig. $3 \mathrm{~b}$ and used for our demonstration in Sec. 3 are subject to error emanating from (a) our manual extraction of adsorption data from plots found in the literature, (b) the lack of adsorption data at extremely low pressures (i.e., possibly inappropriate fitting to adsorption data that is outside the Henry regime), (c) the number of data points we used in the fitting procedure, ${ }^{124}$ and (d) slight differences in temperature at which the adsorption was measured. Third, our demonstration assumed that the gas composition space was two-dimensional- i.e., that no gases other than $\mathrm{CO}_{2}$ and $\mathrm{SO}_{2}$ adsorb in the MOF.

The ideas and intuition generated in our article lay a foundation for future work in sensor array development. (1) Though we focused on $\mathrm{CO}_{2} / \mathrm{SO}_{2}$ sensing for context, it is practically relevant since (a) high concentrations of $\mathrm{CO}_{2}$ and $\mathrm{SO}_{2}$ are detrimental to health and the environment ${ }^{81-83}$ and (b) $\mathrm{CO}_{2}$ and $\mathrm{SO}_{2}$ concentrations in volcanic plume gas are predictive of the imminence of volcanic eruptions. ${ }^{86,87}$ Therefore, we aim to experimentally verify our prediction 
that NOTT-300 and KAUST-7 compose a robust QCM-MOF sensor array for $\mathrm{CO}_{2}$ and $\mathrm{SO}_{2}$ sensing (see Fig. 6), assuming other gas species do not adsorb appreciably. Box 4 discusses the experimental fabrication of MOF thin films on QCMs. (2) Using molecular models, ${ }^{64,72}$ we aim to simulate adsorption of common gas molecules in a large database of computationready, experimental MOF structures; ${ }^{68}$ on the basis of these predicted Henry coefficients, we can apply the framework herein to select the optimal subset to compose robust QCM-based sensor arrays at dilute conditions for more complicated (i.e., more than two components) gas sensing applications. (3) We seek to extend our ideas for choosing subsets of MOFs for robust sensor arrays to the case where the mapping from composition to response space (as in Fig. 4) is non-linear, accounting for (a) saturation and (b) non-independent contributions to adsorption by each gas species. Gustafson and Wilmer ${ }^{65,66}$ properly account for (a) and (b) through the evaluation of MOF-based sensor arrays by conducting a grand-canonical Monte Carlo simulation of adsorption at each gas phase composition/condition to which the MOFs are exposed. However, this comes at greater computational cost compared to our technique in Fig. 6; with measurement of just four Henry coefficients, the 2-array response to all compositions (in the Henry regime) followed. Moreover, we relax the constraint in Gustafson and Wilmer that the total pressure is fixed. Future investigation into how to $\mathrm{cu}-$ rate MOFs for sensor arrays based on a nonlinear, analytical model of adsorption $\mathbf{m}(\mathbf{p})$ would allow for a computation-efficient selection of MOF subsets to compose a robust sensor array while accounting for saturation and non-independent contributions to adsorption by each gas and allowing the total pressure to vary.

We initially embarked on this study with the aim of distinguishing changes in $\mathrm{CO}_{2}$ concentration from variable humidity via a two QCMMOF sensor array. However, we found experimentally reported Henry coefficients of water in $\mathrm{MOFs}^{125}$ to be much larger than those of $\mathrm{CO}_{2}$; water dominated the sensor response (i.e., the ellipses in Fig. 4 were flattened). Moreover, most MOFs would saturate with water in humid conditions, rendering their adsorbed mass insensitive to small changes in the gas phase composition. We conclude that an important research direction is to synthesize hydrophobic MOFs for practical sensing applications. Alternatively, owing to the strong affinity that water has for most MOFs, dehumidification of the sample gas via e.g., a desiccant such silica gel ${ }^{126}$ is likely required prior to exposing the QCMMOF array to the gas for analysis.

We assumed isothermal conditions throughout. However, we can account for the [nonlinear] temperature dependence of the Henry coefficients in eqn. 1 for operation of the sensor array in environments of varying temperature. The temperature $T$ can then be (i) measured and directly input into the model $\mathbf{m}(\mathbf{p} ; T)$ to determine the gas composition $\mathbf{p}$ from the sensor array response $\mathbf{m}$ or (ii) interestingly but perhaps not practically desirable, predicted by the sensor array response (at least $g+1$ sensors are required to determine the concentration of $g$ gases and $T$ ).

The singular value decomposition is a widely useful matrix factorization ${ }^{71}$ with additional uses in gas sensing, including to reduce the dimensionality of sensor array data (SVD is directly related to principal component analysis) ${ }^{52}$ and denoise sensor array responses. ${ }^{127} \mathrm{In}$ control theory, the SVD is used to analyze how the gain in a multi-input, multi-output control system depends on the direction taken in input space, ${ }^{128}$ directly analogous to the anisotropy of the mapping from composition to sensor response space here.

While we focused on coating QCMs with MOFs and gathering information about the gas phase composition by measuring adsorbed mass changes, MOFs can operate as recognition elements through fundamentally different mechanisms. First, flexibility and adsorbate-induced stress on the $\mathrm{MOF}^{123,129,130}$ can be leveraged for gas sensing. For example, Allendorf et al. ${ }^{131}$ deposited a thin film of HKUST-1- a MOF whose unit cell contracts upon dehydration ${ }^{132}$ on the surface of a microcantilever with a builtin piezoresistive sensor to detect adsorptioninduced stress. The electrical resistance in the 
sensor correlated strongly with the concentration of water vapor in nitrogen. Freund et al. recently leveraged the gas-induced large $\leftrightarrow$ narrow pore transition of breathing MOF MIL$53(\mathrm{Al})$ to construct a $\mathrm{CO}_{2}$ sensor. ${ }^{133}$ Second, luminescent $\mathrm{MOFs}^{134}$ can be leveraged for gas sensing by observing shifts in the emission spectra or enhancement/quenching of the intensity induced by adsorbed gas. ${ }^{17}$ Takashima et al. ${ }^{135}$ combined luminescence and flexibility to demonstrate a MOF sensor by synthesizing a MOF (a) composed of two entangled nets that displace upon gas adsorption and (b) that fluoresces at an intensity that is non-linearly related to the concentration of toluene in a helium gas stream. Third, porous, conductive MOFs ${ }^{136}$ whose electrical conductivity is modulated by interactions with gas molecules can be leveraged for gas sensing. For example, Stassen et al. ${ }^{137}$ reported a $2 \mathrm{D}$ MOF whose electrical conductivity is linearly correlated to 400-1000 ppm $\mathrm{CO}_{2}$ concentration in air; water influenced the correlation minimally within a range of 10 $80 \%$ relative humidity. Campbell et al. ${ }^{138}$ constructed a sensor array composed of three conductive MOFs; the response pattern to volatile organic compounds clustered according to alcohols, aromatics, aliphatics, amines, and ketones/esters. In addition to QCMs, MOFs can be coated as thin films on other transducers to compose a gas sensor, including cantilevers ${ }^{33}$ and surface wave acoustic devices. ${ }^{139,140}$

The perspective of a sensor array as a map from gas composition to response space can be adapted to curate materials for stress-, luminescence-, and conductance-based sensor arrays by substituting the mass measurements by the QCMs, m, with stress, optical intensity, or electronic conductivity, respectively. Our material curation methodology throughout then applies if the response (e.g. conductivity) is linearly related to the gas composition, with $\mathbf{H}$ in eqn. 2 containing the coefficients.

A unique advantage of pursuing a sensor array based on QCM-MOFs is that the mass of gas adsorbed in a MOF under a given bulk gas condition (temperature, pressure, composition) is an intrinsic material property that can be reliably predicted a priori via molec- ular modeling and simulation. ${ }^{64}$ This enables the computational curation of MOFs for QCMbased gas sensor arrays, as opposed to the timeand resource-intensive Edisonian approach of synthesizing many MOFs and measuring their response to numerous conditions. Moreover, the number of materials known to exhibit adsorbate-modulated flexibility, luminescence, or conductance is small compared to the number of MOFs known to adsorb gas; the latter lends a greater number of materials from which to draw diverse components for a sensor array of QCM-MOFs.

Acknowledgement C.M.S. thanks the School of Chemical, Biological, and Environmental Engineering (CBEE) at Oregon State University for start-up funds.

Supporting Information Available: The raw data and Jupyter Notebooks with Julia ${ }^{141}$ code used to produce the plots in this article are available on Github at https://github.com/ SimonEnsemble/sensor_arrays. This material is available free of charge via the Internet at http://pubs.acs.org/.

\section{References}

(1) Azad, A.; Akbar, S.; Mhaisalkar, S. et al. Solid-state gas sensors: A review. Journal of the Electrochemical Society 1992, 139, 3690-3704.

(2) Sekhar, P. K.; Brosha, E. L.; Mukundan, R. et al. Chemical sensors for environmental monitoring and homeland security. The Electrochemical Society Interface 2010, 19, 35-40.

(3) Schaller, E.; Bosset, J. O.; Escher, F. Electronic noses and their application to food. LWT-Food Science and Technology 1998, 31, 305-316.

(4) Thompson, J. E. Crowd-sourced air quality studies: A review of the literature \& portable sensors. Trends in Environmental Analytical Chemistry 2016, 11, 2334. 
(5) Lee, D.-D.; Lee, D.-S. Environmental gas sensors. IEEE Sensors Journal 2001, 1, 214-224.

(6) Shirasu, M.; Touhara, K. The scent of disease: volatile organic compounds of the human body related to disease and disorder. The Journal of Biochemistry 2011, 150, 257-266.

(7) Janata, J.; Josowicz, M. Conducting polymers in electronic chemical sensors. Nature Materials 2003, 2, 19.

(8) Bai, H.; Shi, G. Gas sensors based on conducting polymers. Sensors 2007, 7, 267-307.

(9) Rakow, N. A.; Suslick, K. S. A colorimetric sensor array for odour visualization. Nature 2000, 406, 710.

(10) Wang, C.; Yin, L.; Zhang, L. et al. Metal oxide gas sensors: sensitivity and influencing factors. Sensors 2010, 10, 20882106 .

(11) Arshak, K.; Moore, E.; Lyons, G. et al. A review of gas sensors employed in electronic nose applications. Sensor Review 2004, 24, 181-198.

(12) Fine, G. F.; Cavanagh, L. M.; Afonja, A. et al. Metal oxide semi-conductor gas sensors in environmental monitoring. Sensors 2010, 10, 5469-5502.

(13) O’Sullivan, C.; Guilbault, G. Commercial quartz crystal microbalances - theory and applications. Biosensors and Bioelectronics 1999, 14, 663 - 670 .

(14) Zacher, D.; Shekhah, O.; Wöll, C. et al. Thin films of metal-organic frameworks. Chemical Society Reviews 2009, 38, 1418-1429.

(15) Stassen, I.; Burtch, N.; Talin, A. et al. An updated roadmap for the integration of metal-organic frameworks with electronic devices and chemical sensors. Chemical Society Reviews 2017, 46, 3185-3241.
(16) Kreno, L. E.; Leong, K.; Farha, O. K. et al. Metal-organic framework materials as chemical sensors. Chemical Reviews 2011, 112, 1105-1125.

(17) Yi, F.-Y.; Chen, D.; Wu, M.-K. et al. Chemical sensors based on metal-organic frameworks. ChemPlusChem 2016, 81, 675-690.

(18) Furukawa, H.; Cordova, K. E.; O'Keeffe, M. et al. The chemistry and applications of metal-organic frameworks. Science 2013, 341, 1230444.

(19) Li, J.-R.; Kuppler, R. J.; Zhou, H.-C. Selective gas adsorption and separation in metal-organic frameworks. Chemical Society Reviews 2009, 38, 1477-1504.

(20) Sauerbrey, G. Verwendung von Schwingquarzen zur Wägung dünner Schichten und zur Mikrowägung. Zeitschrift für Physik 1959, 155, 206-222.

(21) Ameloot, R.; Stappers, L.; Fransaer, J. et al. Patterned growth of metal-organic framework coatings by electrochemical synthesis. Chemistry of Materials 2009, 21, 2580-2582.

(22) Biemmi, E.; Darga, A.; Stock, N. et al. Direct growth of $\mathrm{Cu}_{3}(\mathrm{BTC})_{2}\left(\mathrm{H}_{2} \mathrm{O}\right)_{3}$. $x \mathrm{H}_{2} \mathrm{O}$ thin films on modified QCM-gold electrodes-Water sorption isotherms. Microporous and Mesoporous Materials 2008, 114, 380-386.

(23) Yamagiwa, H.; Sato, S.; Fukawa, T. et al. Detection of volatile organic compounds by weight-detectable sensors coated with metal-organic frameworks. Scientific Reports 2014, 4, 6247.

(24) Tu, M.; Wannapaiboon, S.; Khaletskaya, K. et al. Engineering ZeoliticImidazolate Framework (ZIF) Thin Film Devices for Selective Detection of Volatile Organic Compounds. Advanced Functional Materials 2015, 25, 4470-4479. 
(25) Tchalala, M.; Bhatt, P.; Chappanda, K. et al. Fluorinated MOF platform for selective removal and sensing of $\mathrm{SO}_{2}$ from flue gas and air. Nature Communications 2019, 10, 1328.

(26) Fang, X.; Wang, L.; He, X. et al. A 3D Calcium Spirobifluorene MetalOrganic Framework: Single-Crystalto-Single-Crystal Transformation and Toluene Detection by a Quartz Crystal Microbalance Sensor. Inorganic Chemistry 2018, 57, 1689-1692.

(27) Kosuru, L.; Bouchaala, A.; Jaber, N. et al. Humidity detection using metal organic framework coated on QCM. Journal of Sensors 2016, 2016.

(28) Tchalala, M. R.; Belmabkhout, Y.; Adil, K. et al. Concurrent Sensing of $\mathrm{CO}_{2}$ and $\mathrm{H}_{2} \mathrm{O}$ from Air Using Ultramicroporous Fluorinated Metal-Organic Frameworks: Effect of Transduction Mechanism on the Sensing Performance. ACS Applied Materials $\&$ Interfaces 2018, 11, 1706-1712.

(29) Si, X.; Jiao, C.; Li, F. et al. High and selective $\mathrm{CO}_{2}$ uptake, $\mathrm{H}_{2}$ storage and methanol sensing on the amine-decorated 12-connected MOF CAU-1. Energy \&6 Environmental Science 2011, 4, 45224527.

(30) Wilson, A. Advances in electronic-nose technologies for the detection of volatile biomarker metabolites in the human breath. Metabolites 2015, 5, 140-163.

(31) Albert, K. J.; Lewis, N. S.; Schauer, C. L. et al. Cross-reactive chemical sensor arrays. Chemical Reviews 2000, 100, 25952626 .

(32) Askim, J. R.; Mahmoudi, M.; Suslick, K. S. Optical sensor arrays for chemical sensing: the optoelectronic nose. Chemical Society Reviews 2013, 42, 8649-8682.
(33) Lang, H. P.; Hegner, M.; Gerber, C. Cantilever array sensors. Materials Today 2005, 8, $30-36$.

(34) Persaud, K.; Dodd, G. Analysis of discrimination mechanisms in the mammalian olfactory system using a model nose. Nature 1982, 299.

(35) Gardner, J. W.; Bartlett, P. N. A brief history of electronic noses. Sensors and Actuators B: Chemical 1994, 18, $210-$ 211.

(36) Mombaerts, P. Seven-Transmembrane Proteins as Odorant and Chemosensory Receptors. Science 1999, 286, 707-711.

(37) Buck, L.; Axel, R. A novel multigene family may encode odorant receptors: a molecular basis for odor recognition. Cell 1991, 65, 175-187.

(38) Gilad, Y.; Lancet, D. Population differences in the human functional olfactory repertoire. Molecular Biology and Evolution 2003, 20, 307-314.

(39) Niimura, Y. Evolutionary dynamics of olfactory receptor genes in chordates: interaction between environments and genomic contents. Hum Genomics 2009, 4, 107-118.

(40) Quignon, P.; Giraud, M.; Rimbault, M. et al. The dog and rat olfactory receptor repertoires. Genome Biology 2005, 6, R83.

(41) Vosshall, L. B.; Wong, A. M.; Axel, R. An olfactory sensory map in the fly brain. Cell 2000, 102, 147-159.

(42) Malnic, B.; Hirono, J.; Sato, T. et al. Combinatorial receptor codes for odors. Cell 1999, 96, 713-723.

(43) Mombaerts, P. Odorant receptor gene choice in olfactory sensory neurons: the one receptor-one neuron hypothesis revisited. Current Opinion in Neurobiology 2004, 14, 31-36. 
(44) Bushdid, C.; Magnasco, M. O.; Vosshall, L. B. et al. Humans can discriminate more than 1 trillion olfactory stimuli. Science 2014, 343, 1370-1372.

(45) Gerkin, R. C.; Castro, J. B. The number of olfactory stimuli that humans can discriminate is still unknown. Elife 2015, 4, e08127.

(46) Meister, M. On the dimensionality of odor space. Elife 2015, 4, e07865.

(47) Nagata, Y.; Takeuchi, N. Measurement of odor threshold by triangle odor bag method. Odor Measurement Review 2003, 118, 118-127.

(48) Tisch, U.; Haick, H. Nanomaterials for cross-reactive sensor arrays. MRS Bulletin 2010, 35, 797-803.

(49) Schaller, E.; Bosset, J. O.; Escher, F. Electronic Noses and Their Application to Food. LWT - Food Science and Technology 1998, 31, $305-316$.

(50) Friedman, J.; Hastie, T.; Tibshirani, R. The Elements of Statistical Learning; Springer Series in Statistics New York, 2001; Vol. 1.

(51) Hines, E.; Llobet, E.; Gardner, J. Electronic noses: a review of signal processing techniques. IEE Proceedings-Circuits, Devices and Systems 1999, 146, 297310.

(52) Jurs, P. C.; Bakken, G.; McClelland, H. Computational methods for the analysis of chemical sensor array data from volatile analytes. Chemical Reviews 2000, 100, 2649-2678.

(53) Kumar, P.; Deep, A.; Kim, K.-H. Metal organic frameworks for sensing applications. TrAC Trends in Analytical Chemistry 2015, 73, 39-53.

(54) Woellner, M.; Hausdorf, S.; Klein, N. et al. Adsorption and Detection of Hazardous Trace Gases by Metal-Organic
Frameworks. Advanced Materials 2018, 30, 1704679 .

(55) Wang, H.; Lustig, W. P.; Li, J. Sensing and capture of toxic and hazardous gases and vapors by metal-organic frameworks. Chemical Society Reviews 2018, 47, 4729-4756.

(56) Moghadam, P. Z.; Li, A.; Wiggin, S. B. et al. Development of a Cambridge Structural Database Subset: A Collection of Metal-Organic Frameworks for Past, Present, and Future. Chemistry of Materials 2017, 29, 2618-2625.

(57) Rowsell, J. L.; Yaghi, O. M. Metalorganic frameworks: a new class of porous materials. Microporous and Mesoporous $\mathrm{Ma}^{-}$ terials 2004, 73, 3 - 14 .

(58) Howarth, A. J.; Liu, Y.; Li, P. et al. Chemical, thermal and mechanical stabilities of metal-organic frameworks. $\mathrm{Na}$ ture Reviews Materials 2016, 1, 15018.

(59) Gu, Z.-Y.; Wang, G.; Yan, X.-P. MOF5 metal-organic framework as sorbent for in-field sampling and preconcentration in combination with thermal desorption GC/MS for determination of atmospheric formaldehyde. Analytical Chemistry 2010, 82, 1365-1370.

(60) Smith, P. A.; Koch, D.; Hook, G. L. et al. Detection of gas-phase chemical warfare agents using field-portable gas chromatography-mass spectrometry systems: instrument and sampling strategy considerations. TrAC Trends in Analytical Chemistry 2004, 23, 296-306.

(61) Leidinger, M.; Rieger, M.; Sauerwald, T. et al. Integrated pre-concentrator gas sensor microsystem for ppb level benzene detection. Sensors and Actuators B: Chemical 2016, 236, 988-996.

(62) Britt, D.; Furukawa, H.; Wang, B. et al. Highly efficient separation of carbon dioxide by a metal-organic framework replete with open metal sites. Pro- 
ceedings of the National Academy of Sciences 2009, 106, 20637-20640.

(63) Wang, J.; Luthey-Schulten, Z. A.; Suslick, K. S. Is the olfactory receptor a metalloprotein? Proceedings of the National Academy of Sciences 2003, 100, 30353039 .

(64) Sturluson, A.; Huynh, M. T.; Kaija, A. et al. The role of molecular modeling \& simulation in the discovery and deployment of metal-organic frameworks for gas storage and separation. ChemRxiv 2019,

(65) Gustafson, J. A.; Wilmer, C. E. Computational design of metal-organic framework arrays for gas sensing: influence of array size and composition on sensor performance. The Journal of Physical Chemistry C 2017, 121, 6033-6038.

(66) Gustafson, J. A.; Wilmer, C. E. Optimizing information content in MOF sensor arrays for analyzing methane-air mixtures. Sensors and Actuators B: Chemical 2018, 267, 483-493.

(67) Wilmer, C. E.; Leaf, M.; Lee, C. Y. et al. Large-scale screening of hypothetical metal-organic frameworks. Nature Chemistry 2011, 4, 83-89.

(68) Chung, Y. G.; Camp, J.; Haranczyk, M. et al. Computation-ready, experimental metal-organic frameworks: A tool to enable high-throughput screening of nanoporous crystals. Chemistry of Materials 2014, 26, 6185-6192.

(69) Gustafson, J. A.; Wilmer, C. E. Intelligent selection of metal-organic framework arrays for methane sensing via genetic algorithms. ACS Sensors 2019,

(70) Pearce, T. C. Odor to sensor space transformations in biological and artificial noses. Neurocomputing 2000, 32, 941952.

(71) Strang, G. Introduction to Linear Algebra; Wellesley-Cambridge Press Wellesley, MA, 2016; Vol. 5.
(72) Frenkel, D.; Smit, B. Understanding Molecular Simulation: from Algorithms to Applications; Elsevier, 2001; Vol. 1.

(73) Konvalina, G.; Haick, H. Sensors for breath testing: from nanomaterials to comprehensive disease detection. Accounts of Chemical Research 2013, 47, 66-76.

(74) Hakim, M.; Broza, Y. Y.; Barash, O. et al. Volatile organic compounds of lung cancer and possible biochemical pathways. Chemical Reviews 2012, 112, 5949-5966.

(75) Peng, G.; Hakim, M.; Broza, Y. et al. Detection of lung, breast, colorectal, and prostate cancers from exhaled breath using a single array of nanosensors. British Journal of Cancer 2010, 103, 542.

(76) Machado, R. F.; Laskowski, D.; Deffenderfer, O. et al. Detection of lung cancer by sensor array analyses of exhaled breath. American Journal of Respiratory and Critical Care Medicine 2005, 171, 1286-1291.

(77) Kalman, D. A singularly valuable decomposition: the SVD of a matrix. The College Mathematics Journal 1996, 27, 223.

(78) Smith, S. J.; Aardenne, J. v.; Klimont, Z. et al. Anthropogenic sulfur dioxide emissions: 1850-2005. Atmospheric Chemistry and Physics 2011, 11, 1101-1116.

(79) Le Quéré, C.; Andrew, R. M.; Friedlingstein, P. et al. Global carbon budget 2018. Earth System Science Data (Online) 2018, 10 .

(80) Collins, M.; Knutti, R.; Arblaster, J. et al. In Climate Change 2013: The Physical Science Basis. Contribution of Working Group I to the Fifth Assessment Report of the Intergovernmental Panel on Climate Change; Stocker, T., Qin, D., Plattner, G.-K. et al. , Eds.; Cambridge University Press: Cambridge, 
United Kingdom and New York, NY, USA, 2013; Chapter 12, pp 1029-1136.

(81) Council, N. R. Acute Exposure Guideline Levels for Selected Airborne Chemicals: Volume 8; The National Academies Press: Washington, DC, 2010.

(82) Dincer, I. Renewable energy and sustainable development: a crucial review. Renewable and Sustainable Energy Reviews 2000, 4, 157-175.

(83) Langford, N. J. Carbon dioxide poisoning. Toxicological Reviews 2005, 24, 229235 .

(84) Thordarson, T.; Self, S.; Oskarsson, N. et al. Sulfur, chlorine, and fluorine degassing and atmospheric loading by the 1783-1784 AD Laki (Skaftár Fires) eruption in Iceland. Bulletin of Volcanology 1996, 58, 205-225.

(85) Thordarson, T.; Self, S. Atmospheric and environmental effects of the 1783-1784 Laki eruption: A review and reassessment. Journal of Geophysical Research: Atmospheres 2003, 108.

(86) Aiuppa, A.; Moretti, R.; Federico, C. et al. Forecasting Etna eruptions by realtime observation of volcanic gas composition. Geology 2007, 35, 1115-1118.

(87) Aiuppa, A.; Federico, C.; Giudice, G. et al. The 2007 eruption of Stromboli volcano: insights from real-time measurement of the volcanic gas plume $\mathrm{CO}_{2} / \mathrm{SO}_{2}$ ratio. Journal of Volcanology and Geothermal Research 2009, 182, 221-230.

(88) Beauvais, L. G.; Long, J. R. $\mathrm{Co}_{3}\left[\mathrm{Co}(\mathrm{CN})_{5}\right]_{2}$ : A microporous magnet with an ordering temperature of 38 K. Journal of the American Chemical Society 2002, 124, 12096-12097.

(89) Bhatt, P. M.; Belmabkhout, Y.; Cadiau, A. et al. A fine-tuned fluorinated MOF addresses the needs for trace $\mathrm{CO}_{2}$ removal and air capture using physisorption. Journal of the American Chemical Society 2016, 138, 9301-9307.

(90) Carter, J. H.; Han, X.; Moreau, F. Y. et al. Exceptional Adsorption and Binding of Sulfur Dioxide in a Robust Zirconium-Based Metal-Organic Framework. Journal of the American Chemical Society 2018, 140, 15564-15567.

(91) Yang, S.; Lin, X.; Lewis, W. et al. A partially interpenetrated metal-organic framework for selective hysteretic sorption of carbon dioxide. Nature Materials 2012, 11, 710 .

(92) Dybtsev, D. N.; Chun, H.; Kim, K. Rigid and Flexible: A Highly Porous MetalOrganic Framework with Unusual GuestDependent Dynamic Behavior. Angewandte Chemie International Edition 2004, 43, 5033-5036.

(93) Caskey, S. R.; Wong-Foy, A. G.; Matzger, A. J. Dramatic tuning of carbon dioxide uptake via metal substitution in a coordination polymer with cylindrical pores. Journal of the American Chemical Society 2008, 130, 1087010871.

(94) Kaye, S. S.; Long, J. R. Hydrogen Storage in the Dehydrated Prussian Blue Analogues $\mathrm{M}_{3}\left[\mathrm{Co}(\mathrm{CN})_{6}\right]_{2}(\mathrm{M}=\mathrm{Mn}, \mathrm{Fe}$, $\mathrm{Co}, \mathrm{Ni}, \mathrm{Cu}, \mathrm{Zn})$. Journal of the American Chemical Society 2005, 127, 6506-6507.

(95) Cadiau, A.; Belmabkhout, Y.; Adil, K. et al. Hydrolytically stable fluorinated metal-organic frameworks for energyefficient dehydration. Science 2017, 356, 731-735.

(96) Yang, S.; Sun, J.; Ramirez-Cuesta, A. J. et al. Selectivity and direct visualization of carbon dioxide and sulfur dioxide in a decorated porous host. Nature Chemistry 2012, 4, 887.

(97) Arstad, B.; Fjellvåg, H.; Kongshaug, K. O. et al. Amine functionalised 
metal organic frameworks (MOFs) as adsorbents for carbon dioxide. Adsorption 2008, 14, 755-762.

(98) Qian, J.; Jiang, F.; Yuan, D. et al. Highly selective carbon dioxide adsorption in a water-stable indium-organic framework material. Chemical Communications 2012, 48, 9696-9698.

(99) Liang, Z.; Marshall, M.; Chaffee, A. L. $\mathrm{CO}_{2}$ adsorption, selectivity and water tolerance of pillared-layer metal organic frameworks. Microporous and Mesoporous Materials 2010, 132, 305310.

(100) Chun, H.; Dybtsev, D. N.; Kim, H. et al. Synthesis, X-ray crystal structures, and gas sorption properties of pillared square grid nets based on paddle-wheel motifs: Implications for hydrogen storage in porous materials. Chemistry-A European Journal 2005, 11, 3521-3529.

(101) Yang, S.; Liu, L.; Sun, J. et al. Irreversible network transformation in a dynamic porous host catalyzed by sulfur dioxide. Journal of the American Chemical Society 2013, 135, 4954-4957.

(102) Tan, K.; Canepa, P.; Gong, Q. et al. Mechanism of preferential adsorption of $\mathrm{SO}_{2}$ into two microporous paddle wheel frameworks M(bdc)(ted) 0.5 . Chemistry of Materials 2013, 25, 4653-4662.

(103) Mason, J. A.; Sumida, K.; Herm, Z. R. et al. Evaluating metal-organic frameworks for post-combustion carbon dioxide capture via temperature swing adsorption. Energy \& Environmental Science 2011, 4, 3030-3040.

(104) Savage, M.; Cheng, Y.; Easun, T. L. et al. Selective adsorption of sulfur dioxide in a robust metal-organic framework material. Advanced Materials 2016, 28, 87058711.
(105) Thallapally, P. K.; Motkuri, R. K.; Fernandez, C. A. et al. Prussian blue analogues for $\mathrm{CO}_{2}$ and $\mathrm{SO}_{2}$ capture and separation applications. Inorganic Chemistry 2010, 49, 4909-4915.

(106) Wegert, E.; Semmler, G. Phase plots of complex functions: a journey in illustration. Notices AMS 2010, 58, 768-780.

(107) Thompson, R. The behavior of eigenvalues and singular values under perturbations of restricted rank. Linear Algebra and its Applications 1976, 13, 69-78.

(108) Ruthven, D.; Loughlin, K.; Holborow, K. Multicomponent sorption equilibrium in molecular sieve zeolites. Chemical Engineering Science 1973, 28, 701-709.

(109) Heinke, L.; Gu, Z.; Wöll, C. The surface barrier phenomenon at the loading of metal-organic frameworks. Nature Communications 2014, 5, 4562.

(110) Wannapaiboon, S.; Tu, M.; Sumida, K. et al. Hierarchical structuring of metalorganic framework thin-films on quartz crystal microbalance (QCM) substrates for selective adsorption applications. Journal of Materials Chemistry A 2015, 3, 23385-23394.

(111) Wannapaiboon, S.; Tu, M.; Fischer, R. A. Liquid Phase Heteroepitaxial Growth of Moisture-Tolerant MOF-5 Isotype Thin Films and Assessment of the Sorption Properties by Quartz Crystal Microbalance. Advanced Functional Materials 2014, 24, 2696-2705.

(112) Tu, M.; Fischer, R. A. Heteroepitaxial growth of surface mounted metal-organic framework thin films with hybrid adsorption functionality. Journal of Materials Chemistry A 2014, 2, 2018-2022.

(113) Heinke, L.; Wöll, C. Adsorption and diffusion in thin films of nanoporous metalorganic frameworks: ferrocene in SURMOF $\mathrm{Cu}_{2}$ (ndc) $)_{2}$ (dabco). Physical Chem- 
istry Chemical Physics 2013, 15, 92959299.

(114) Liu, B.; Tu, M.; Fischer, R. A. Metalorganic framework thin films: crystallite orientation dependent adsorption. Angewandte Chemie International Edition 2013, 52, 3402-3405.

(115) Stavila, V.; Volponi, J.; Katzenmeyer, A. M. et al. Kinetics and mechanism of metal-organic framework thin film growth: systematic investigation of HKUST-1 deposition on QCM electrodes. Chemical Science 2012, 3, $1531-1540$.

(116) Zacher, D.; Yusenko, K.; Bétard, A. et al. Liquid-Phase Epitaxy of Multicomponent Layer-Based Porous Coordination Polymer Thin Films of $\left[\mathrm{M}(\mathrm{L})(\mathrm{P})_{0.5}\right]$ Type: Importance of Deposition Sequence on the Oriented Growth. Chemistry-A European Journal 2011, 17, 1448-1455.

(117) Zybaylo, O.; Shekhah, O.; Wang, H. et al. A novel method to measure diffusion coefficients in porous metal-organic frameworks. Physical Chemistry Chemical Physics 2010, 12, 8093-8098.

(118) Shekhah, O.; Wang, H.; Kowarik, S. et al. Step-by-step route for the synthesis of metal-organic frameworks. Journal of the American Chemical Society 2007, 129, 15118-15119.

(119) Nijem, N.; Fursich, K.; Bluhm, H. et al. Ammonia adsorption and co-adsorption with water in HKUST-1: spectroscopic evidence for cooperative interactions. The Journal of Physical Chemistry $C$ 2015, 119, 24781-24788.

(120) McDonald, T. M.; Mason, J. A.; Kong, X. et al. Cooperative insertion of $\mathrm{CO}_{2}$ in diamine-appended metal-organic frameworks. Nature 2015, 519, 303.

(121) Walton, K. S.; Millward, A. R.; Dubbeldam, D. et al. Understanding inflections and steps in carbon dioxide adsorption isotherms in metal-organic frameworks. Journal of the American Chemical Society 2008, 130, 406-407.

(122) Elsaidi, S. K.; Mohamed, M. H.; Simon, C. M. et al. Effect of ring rotation upon gas adsorption in SIFSIX-3$\mathrm{M}(\mathrm{M}=\mathrm{Fe}, \mathrm{Ni})$ pillared square grid networks. Chemical Science 2017, 8, 23732380 .

(123) Horike, S.; Shimomura, S.; Kitagawa, S. Soft porous crystals. Nature Chemistry 2009, 1, 695 .

(124) Monpezat, A.; Topin, S.; Deliere, L. et al. Evaluation methods of adsorbents for air purification and gas separation at low concentration. Case studies on xenon and krypton. Industrial \& Engineering Chemistry Research 2019,

(125) Canivet, J.; Bonnefoy, J.; Daniel, C. et al. Structure-property relationships of water adsorption in metal-organic frameworks. New Journal of Chemistry 2014, 38, 3102-3111.

(126) Nishibori, M.; Shin, W.; Izu, N. et al. Sensing performance of thermoelectric hydrogen sensor for breath hydrogen analysis. Sensors and Actuators B: Chemical 2009, 137, 524-528.

(127) Jha, S. K.; Yadava, R. Denoising by singular value decomposition and its application to electronic nose data processing. IEEE Sensors Journal 2011, 11, 35-44.

(128) Andersen, H. W.; Kümmel, M. Evaluating estimation of gain directionality: Part 1: Methodology. Journal of Process Control 1992, 2, 59-66.

(129) Coudert, F.-X. Responsive metalorganic frameworks and framework materials: under pressure, taking the heat, in the spotlight, with friends. Chemistry of Materials 2015, 27, 1905-1916. 
(130) Schneemann, A.; Bon, V.; Schwedler, I. et al. Flexible metal-organic frameworks. Chemical Society Reviews 2014, 43, 6062-6096.

(131) Allendorf, M. D.; Houk, R. J.; Andruszkiewicz, L. et al. Stress-induced chemical detection using flexible metalorganic frameworks. Journal of the American Chemical Society 2008, 130, 14404-14405.

(132) Prestipino, C.; Regli, L.; Vitillo, J. G. et al. Local structure of framework $\mathrm{Cu}(\mathrm{II})$ in HKUST-1 metallorganic framework: spectroscopic characterization upon activation and interaction with adsorbates. Chemistry of Materials 2006, 18, 1337-1346.

(133) Freund, P.; Mielewczyk, L.; Rauche, M. et al. MIL-53(Al)/Carbon Films for $\mathrm{CO}_{2}{ }^{-}$ Sensing at High Pressure. ACS Sustainable Chemistry \&f Engineering 2019, 7, 4012-4018.

(134) Allendorf, M.; Bauer, C.; Bhakta, R. et al. Luminescent metal-organic frameworks. Chemical Society Reviews 2009, 38, 1330-1352.

(135) Takashima, Y.; Martínez, V. M.; Furukawa, S. et al. Molecular decoding using luminescence from an entangled porous framework. Nature Communications 2011, 2, 168.

(136) Sun, L.; Campbell, M. G.; Dincă, M. Electrically conductive porous metalorganic frameworks. Angewandte Chemie International Edition 2016, 55, 35663579 .

(137) Stassen, I.; Dou, J.-H.; Hendon, C. et al. Chemiresistive Sensing of Ambient $\mathrm{CO}_{2}$ by an Autogenously Hydrated $\mathrm{Cu}_{3}$ (hexaiminobenzene) ${ }_{2}$ Framework. ACS Central Science 2019,

(138) Campbell, M. G.; Liu, S. F.; Swager, T. M. et al. Chemiresistive sensor arrays from conductive 2D metal-organic frameworks. Journal of the American Chemical Society 2015, 137, 1378013783.

(139) Grate, J. W. Acoustic wave microsensor arrays for vapor sensing. Chemical Reviews 2000, 100, 2627-2648.

(140) Robinson, A. L.; Stavila, V.; Zeitler, T. R. et al. Ultrasensitive humidity detection using metal-organic framework-coated microsensors. Analytical Chemistry 2012, 84, 7043-7051.

(141) Bezanson, J.; Edelman, A.; Karpinski, S. et al. Julia: A fresh approach to numerical computing. SIAM Review 2017, 59, 65-98. 


\section{Curating metal-organic frameworks to compose robust gas sensor arrays in dilute conditions}

Arni Sturluson, Rachel Sousa, Yujing Zhang, Melanie T. Huynh, Caleb Laird, Arthur H. P. York, Carson Silsby, Chih-Hung Chang, and Cory M. Simon*

Oregon State University

School of Chemical, Biological, and Environmental Engineering. Corvallis, OR, USA

E-mail: Cory.Simon@oregonstate.edu

\section{S1 Identifying Henry coefficients from experimental adsorption isotherms}

According to Henry's law, the equilibrium adsorption of a gas species is proportional to its partial pressure:

$$
m=H p .
$$

Here, $m$ is the mass of gas adsorbed in the adsorbent, $H$ is the Henry coefficient of the adsorbate in the adsorbent [mass of gas/(mass of material-bar)] and $p$ is the partial pressure of the adsorbate in the gas phase [bar]. Henry's law assumes infinite dilution, where there are no interactions between gas molecules, and the only interactions accounted for are between the adsorbate and adsorbent. This assumption can be limiting, but is valid at low pressures - in the Henry regime - allowing us to estimate Henry coefficients by finding the initial $\left(p \rightarrow 0^{+}\right)$slope of adsorption isotherms by fitting to low-pressure adsorption data.

The $\mathrm{CO}_{2}$ and $\mathrm{SO}_{2}$ experimental adsorption isotherms in the MOFs in Fig. 3a were ex- 
tracted from literature ${ }^{1-9}$ using g3data Graph Analyzer. With $12 \mathrm{CO}_{2}$ adsorption isotherms and $12 \mathrm{SO}_{2}$ adsorption isotherms, the number of points chosen to fit the Henry coefficient to were manually chosen (see Jupyter notebooks on https://github.com/SimonEnsemble/ sensor_arrays) rather than automatically because the number of data points in the Henry regime for each adsorption isotherm might differ. We searched for adsorption isotherms in the range of 293 - $298 \mathrm{~K}$. This method is not without deficiencies, as errors are introduced in a few steps in this process: (1) errors associated with the original experiment, (2) errors in the isotherm extraction using g3data, (3) errors in the fitting procedure by (possibly) inappropriately fitting to data outside the Henry regime and (4) slight temperature differences among the measurements.

The Henry coefficient fitting procedure involved using a linear least squares method, as implemented by the MultivariateStats.jl package in Julia, where the mean squared error between the line described by eqn. $\mathrm{S} 1$ and the data points is minimized by varying $H$. For the procedure, the intercept of the line is constrained to be at the origin. Figs. S1-S11 show the result of the Henry coefficient fitting procedure for all the adsorption isotherms. The orange points show the adsorption isotherm data obtained from the literature. The filled squares where used in the Henry coefficient fitting procedure, while the hollow squares were not used in the fitting procedure. The red dashed line shows the resulting Henry's law in eqn. S1 from the identified Henry coefficient $H$; the Henry's law model is extended past the Henry regime, highlighting how poor of an approximation Henry's law is outside of the Henry regime (e.g., Figs. S3a, S6b, S7b). To see the Henry coefficient fit more clearly, a zoomed-in version of the graph near the origin is shown in the inset on the bottom right of each figure.

The resulting spread and correlation of Henry coefficients identified from these adsorption isotherms are depicted in Fig. 3b. 


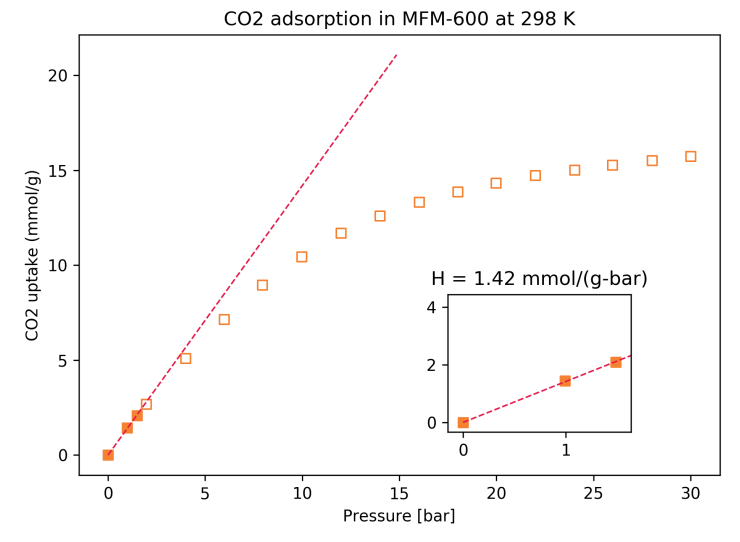

(a)

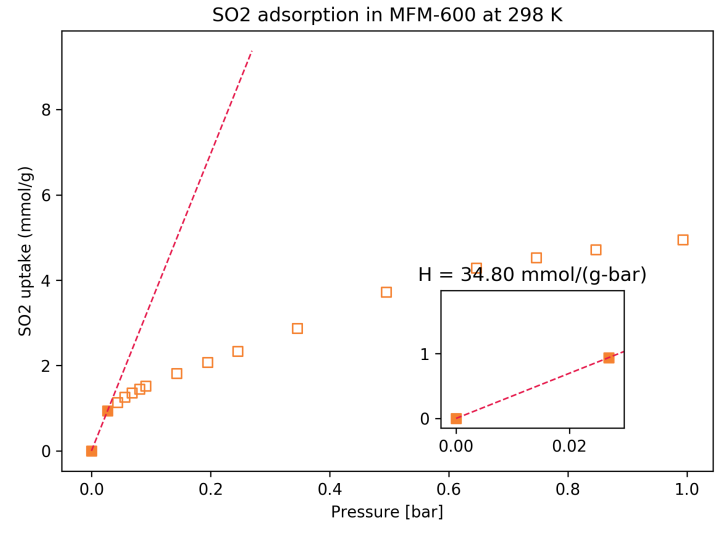

(b)

Figure S1: The Henry extraction of the (a) $\mathrm{CO}_{2}$ and (b) $\mathrm{SO}_{2}$ adsorption isotherms of MFM$600 .^{2}$

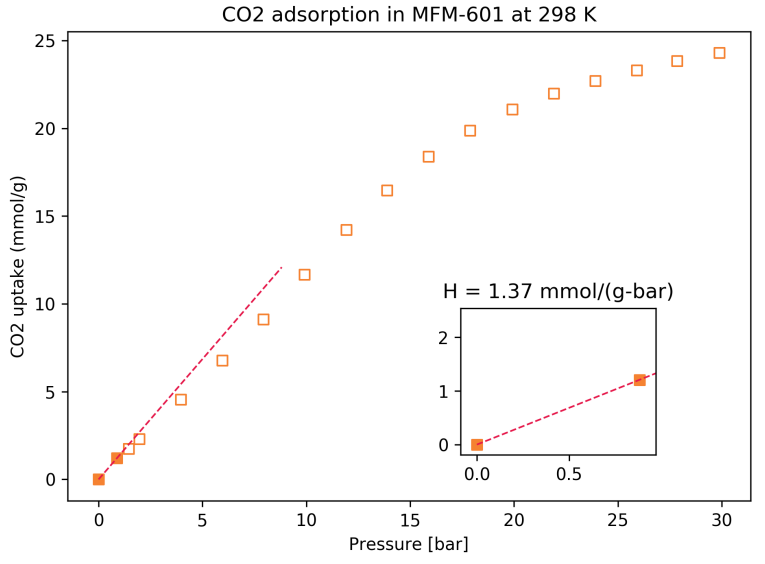

(a)

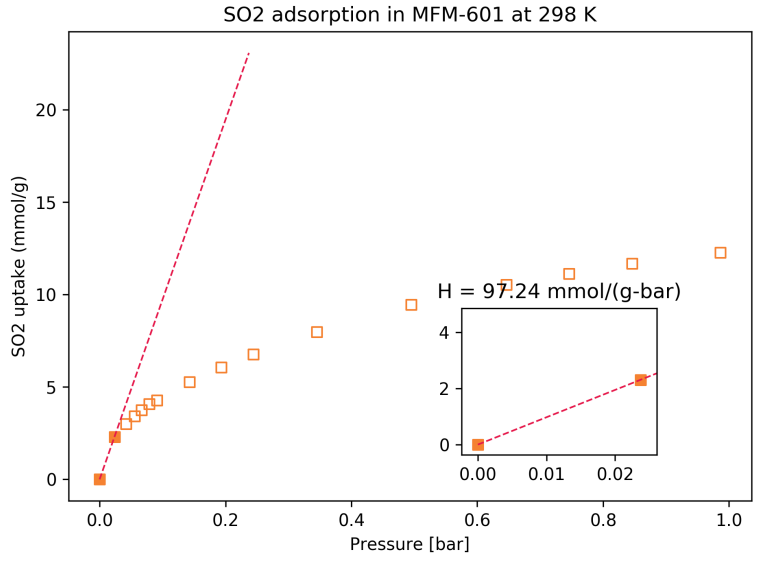

(b)

Figure S2: The Henry extraction of the (a) $\mathrm{CO}_{2}$ and (b) $\mathrm{SO}_{2}$ adsorption isotherms of MFM$601 .^{2}$ 


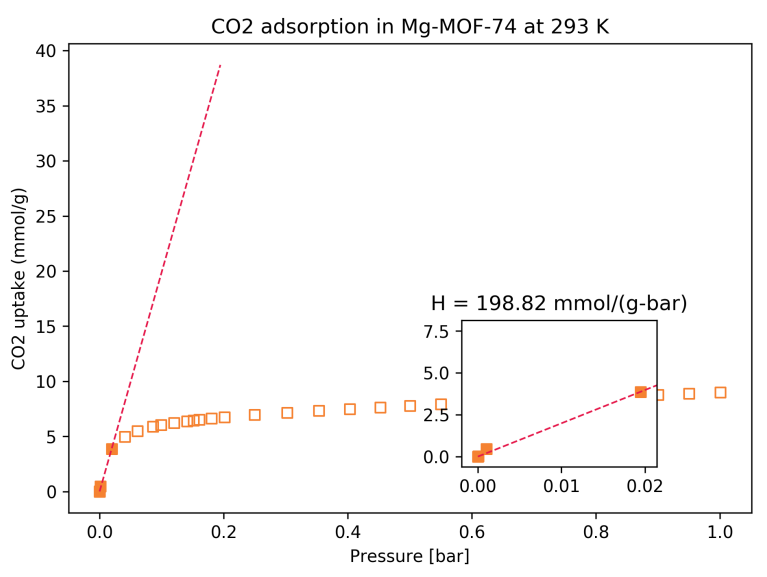

(a)

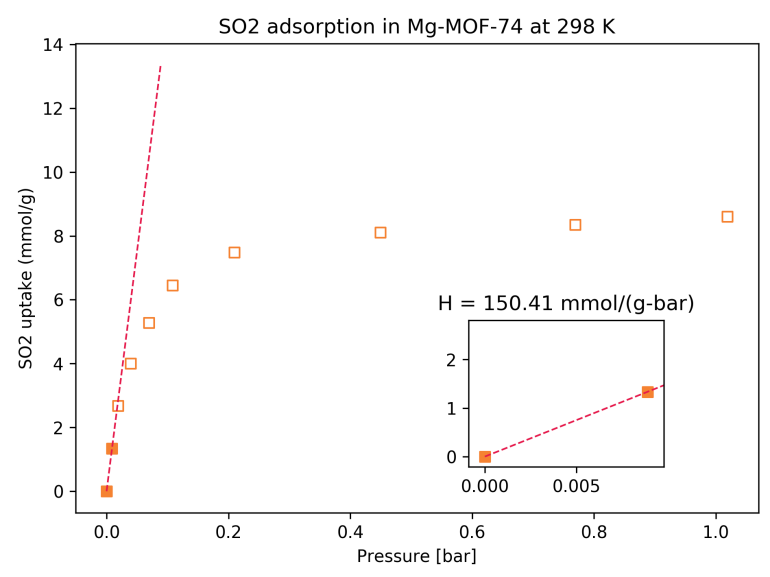

(b)

Figure S3: The Henry extraction of the (a) $\mathrm{CO}_{2}$ and (b) $\mathrm{SO}_{2}$ adsorption isotherms of $\mathrm{Mg}$ MOF-74. ${ }^{3,4}$

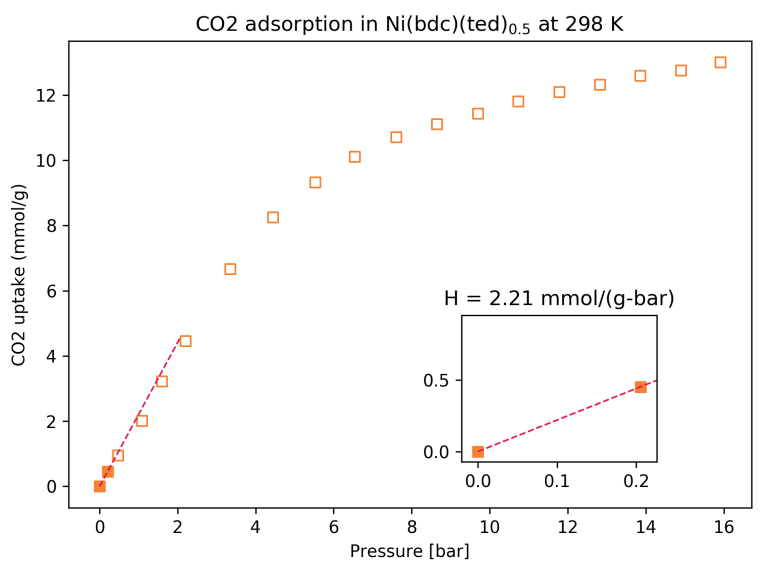

(a)

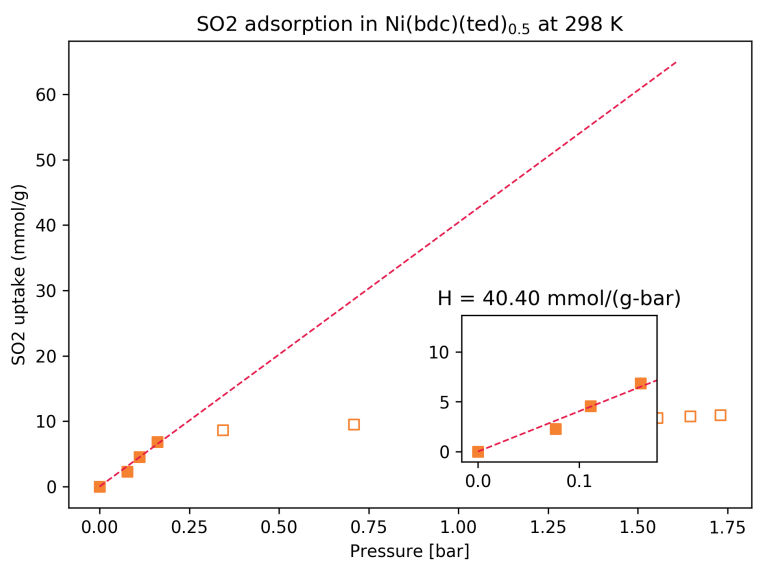

(b)

Figure S4: The Henry extraction of the (a) $\mathrm{CO}_{2}$ and (b) $\mathrm{SO}_{2}$ adsorption isotherms of $\mathrm{Ni}(\mathrm{bdc})(\text { ted })_{0.5} \cdot{ }^{3,5}$ 


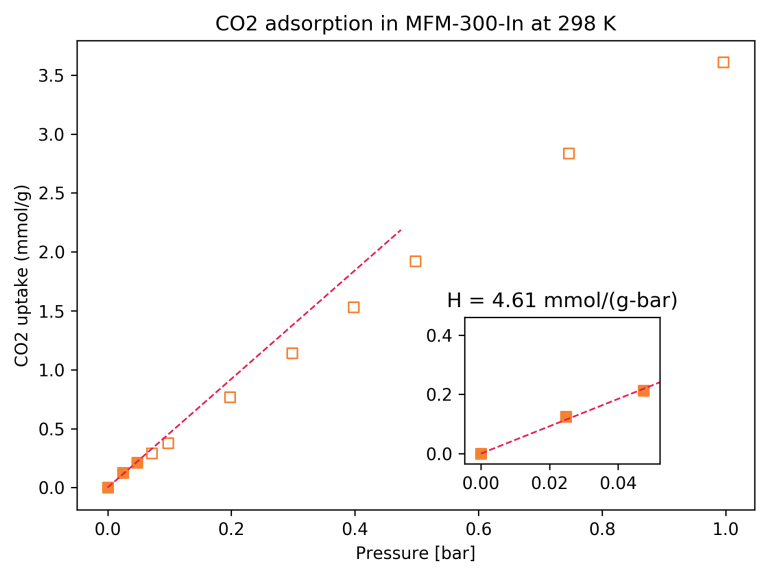

(a)

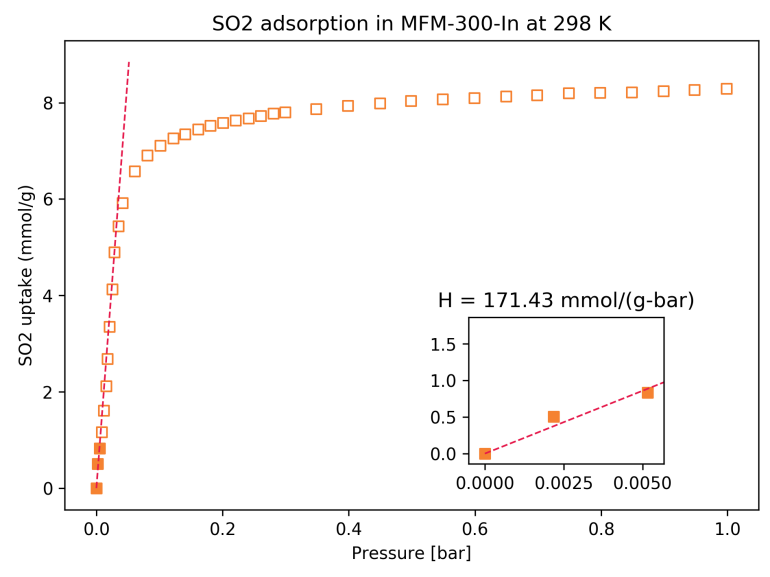

(b)

Figure S5: The Henry extraction of the (a) $\mathrm{CO}_{2}$ and (b) $\mathrm{SO}_{2}$ adsorption isotherms of MFM300(In). ${ }^{5,6}$

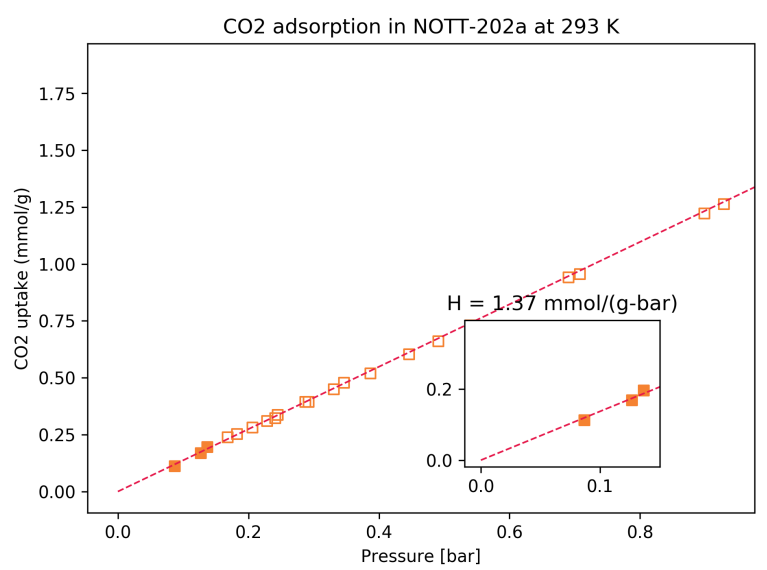

(a)

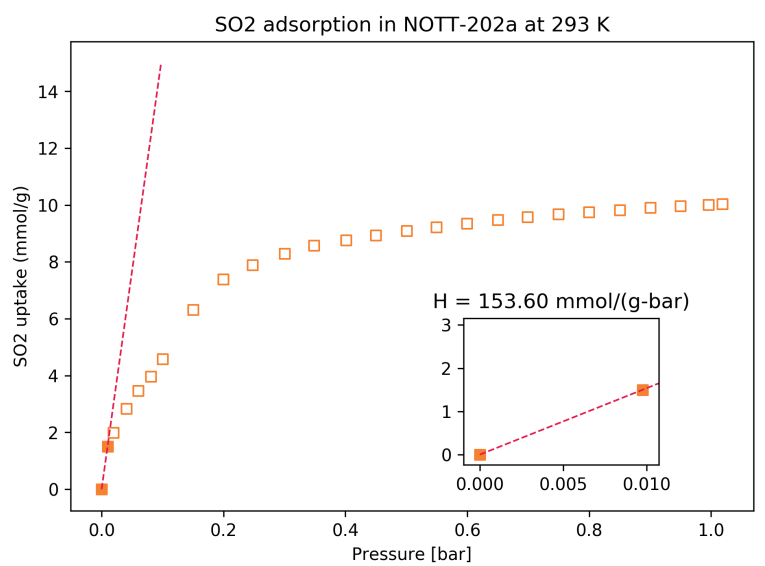

(b)

Figure S6: The Henry extraction of the (a) $\mathrm{CO}_{2}$ and (b) $\mathrm{SO}_{2}$ adsorption isotherms of NOTT202a. ${ }^{7}$ 


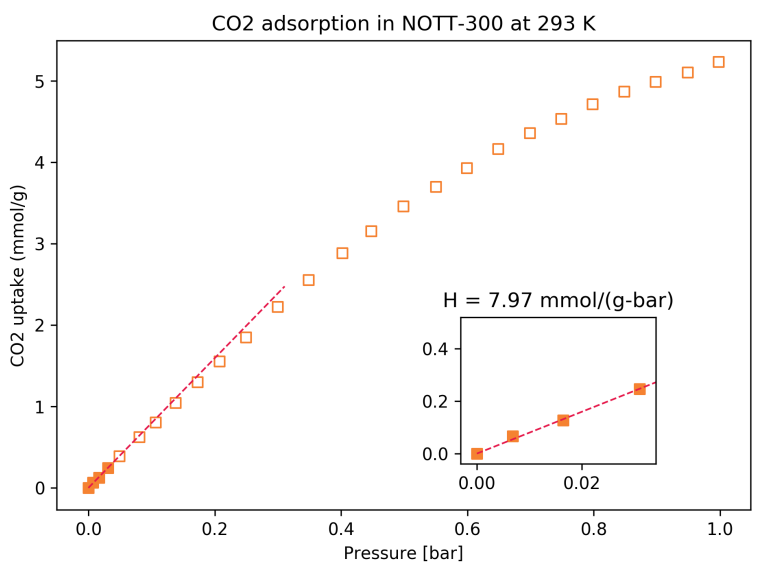

(a)

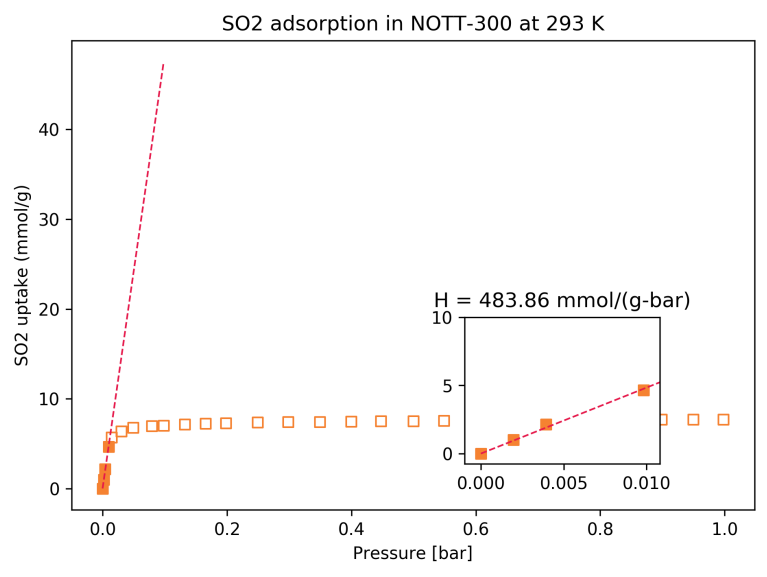

(b)

Figure S7: The Henry extraction of the (a) $\mathrm{CO}_{2}$ and (b) $\mathrm{SO}_{2}$ adsorption isotherms of NOTT$300 .^{8}$

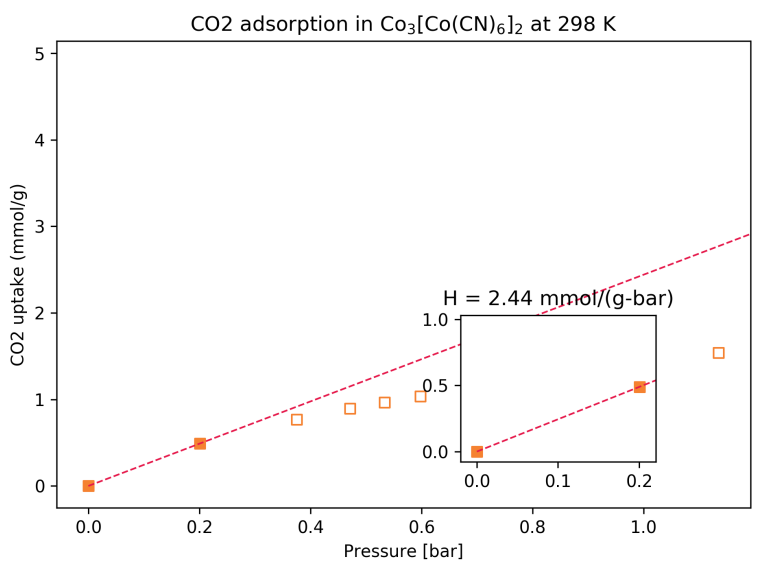

(a)

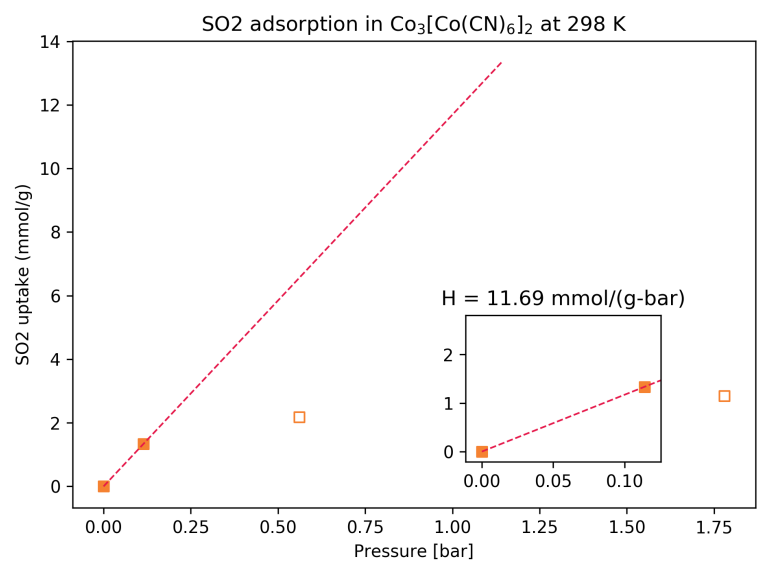

(b)

Figure S8: The Henry extraction of the (a) $\mathrm{CO}_{2}$ and (b) $\mathrm{SO}_{2}$ adsorption isotherms of $\mathrm{Co}_{3}\left[\mathrm{Co}(\mathrm{CN})_{6}\right]_{2} \cdot{ }^{9}$ 


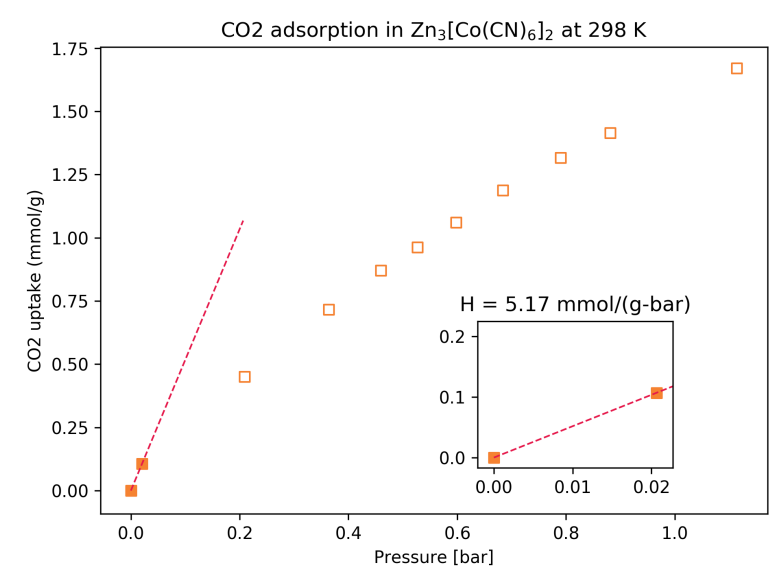

(a)

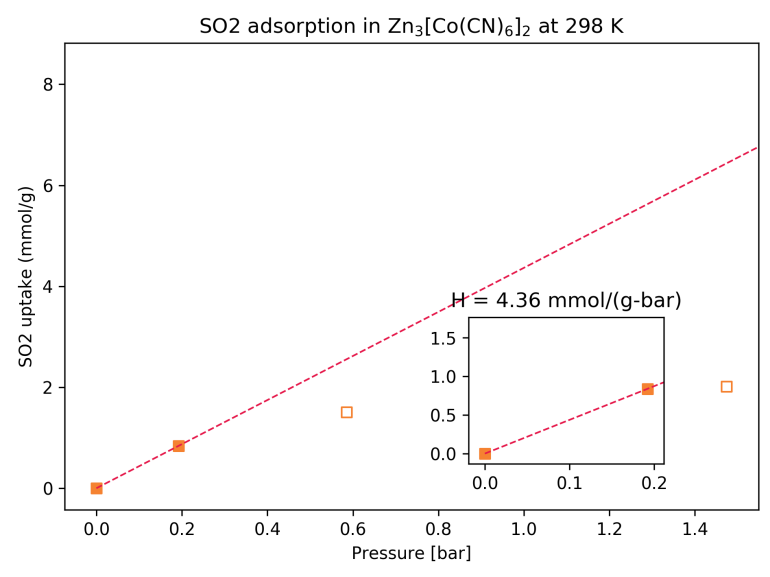

(b)

Figure S9: The Henry extraction of the (a) $\mathrm{CO}_{2}$ and (b) $\mathrm{SO}_{2}$ adsorption isotherms of $\mathrm{Zn}_{3}\left[\mathrm{Co}(\mathrm{CN})_{6}\right]_{2} \cdot{ }^{9}$

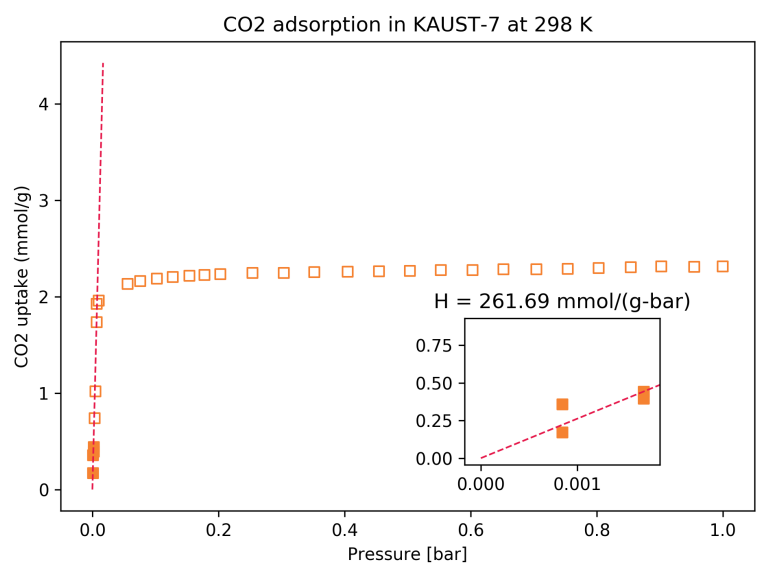

(a)

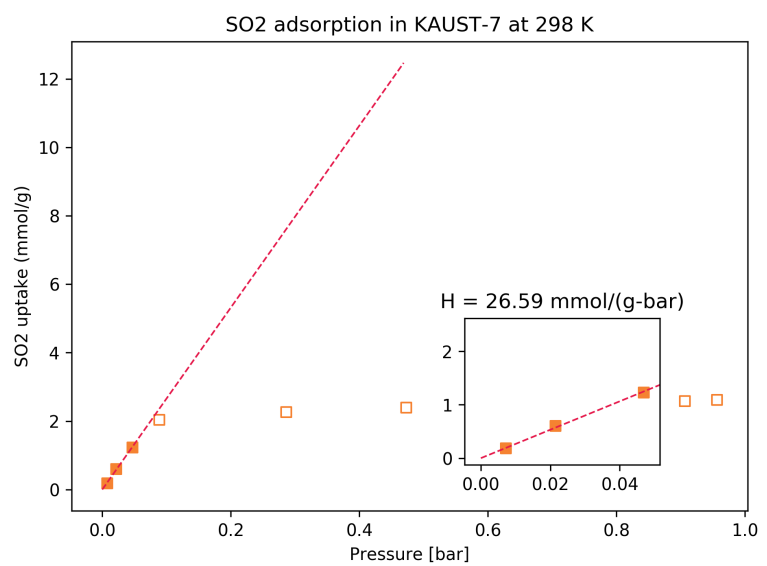

(b)

Figure S10: The Henry extraction of the (a) $\mathrm{CO}_{2}$ and (b) $\mathrm{SO}_{2}$ adsorption isotherms of KAUST-7. ${ }^{1}$ 


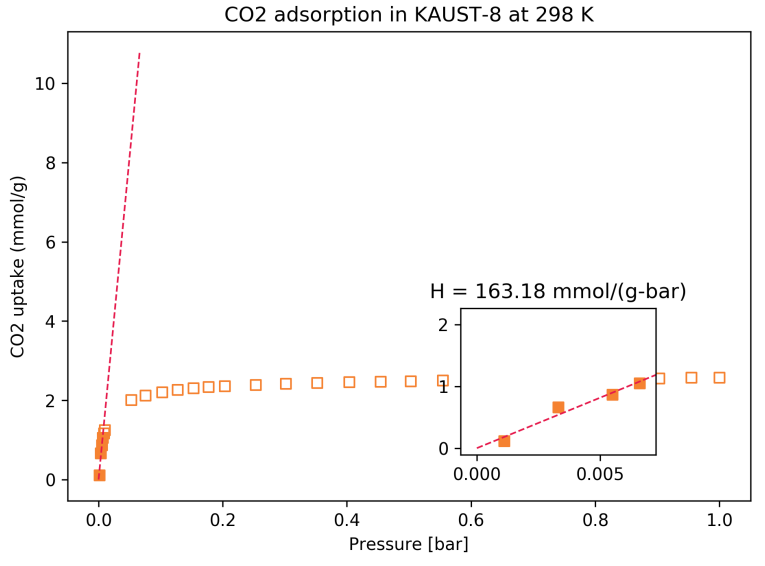

(a)

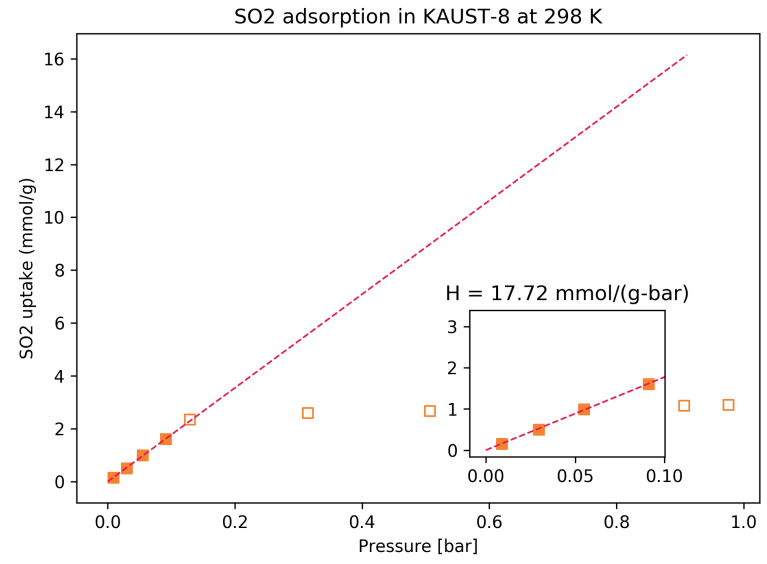

(b)

Figure S11: The Henry extraction of the (a) $\mathrm{CO}_{2}$ and (b) $\mathrm{SO}_{2}$ adsorption isotherms of KAUST-8. ${ }^{1}$ 


\section{S2 On the [inadequate] strategy to curate MOFs for sensor arrays based on their adsorptive selectivity}

Based on [fallacious] intuition, one may propose the following strategy to select the optimal subset of MOFs for a QCM-based gas sensor array: from a large set of MOF candidates, select, for the $i$ th MOF in the sensor array, the MOF that exhibits the highest adsorptive selectivity for gas $i$, for $i=1,2, \ldots, g$ with $g$ the number of gases. If this proposition were true, it would be unnecessary to compute the singular value decomposition of $\mathbf{H}$ for every combination of MOFs to assess the fitness of each combination; instead, we could simply search for the MOFs exhibiting the highest selectivities for the gases that constitute the mixture. However, we reject this strategy to simply choose the MOFs with the highest adsorptive selectivity by providing two hypothetical case examples in Fig. S12 where the strategy fails to recover the most robust and accurate sensor array. Moreover, it is unclear how this strategy would extend to the overdetermined scenario where we have more sensors than gases $(n>g)$.

For simplicity, we consider the objective to curate $n=2$ MOFs for a sensor array of two QCM-MOFs to determine the composition of a gas mixture with $g=2$ components, $A$ and $B$. Each panel in Fig. S12 displays the Henry coefficients of gases $A$ and $B, H_{A}$ and $H_{B}$ respectively, in (a) three and (b) six MOFs serving as candidates for the sensor array, analogous to Fig 3b. One can gauge the $B / A$ selectivity $H_{B} / H_{A}$ of each MOF from the plot via the slope of the line passing through the origin and the point representing the MOF. In Fig. S12a, MOF-1 offers the highest $B / A$ selectivity, whereas MOF-2 offers the highest $A / B$ selectivity. Therefore, the strategy to select the two MOFs with the highest $A / B$ and $B / A$ selectivity would suggest that MOF-1 and MOF-2 would form the most robust and accurate QCM-MOF sensor array. However, the pair composed of MOF-2 and MOF-3

yields the most robust and accurate sensor array, since it yields the largest $\sigma_{2}(\mathbf{H})$. Thus, the aforementioned strategy failed to identify the optimal pair of MOFs from these three 
candidates. In Fig. S12b, MOF-1 offers the highest $B / A$ selectivity and MOF- 5 offers the highest $A / B$ selectivity. Therefore, the strategy to select the two MOFs with the highest $A / B$ and $B / A$ selectivity would suggest that MOF-1 and MOF-5 would form the most robust and accurate QCM-MOF sensor array. However, the pair composed of MOF-4 and MOF-6 yields the most robust and accurate sensor array, since it yields the largest $\sigma_{2}(\mathbf{H})$. Thus, the aforementioned strategy failed to identify the optimal pair of MOFs from these six candidates; neither of the two MOFs exhibiting the highest $B / A$ and $A / B$ selectivity belong to the optimal sensor array.

We conclude that the singular value decomposition of the Henry coefficient matrix $\mathbf{H}$ is necessary to identify the optimal subset of MOFs to place in a robustly accurate QCMMOF sensor array; i.e., curation heuristics based on adsorptive selectivity are suboptimal for curating MOFs for sensor arrays. The reason that we cannot consider adsorptive selectivity alone is that the magnitude of the Henry coefficients must be considered as well; a large Henry coefficient equips a MOF with high sensitivity to changes in the gas composition. Referring to Fig. 5, large Henry coefficients enable the sensor array response to escape the error zone, in which the response is indistinguishable from noise/error, for small changes in gas composition. 


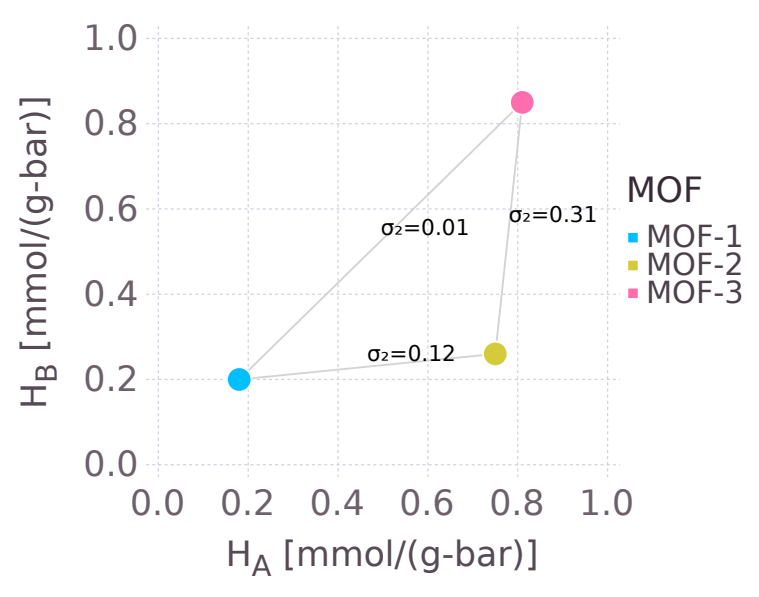

(a)

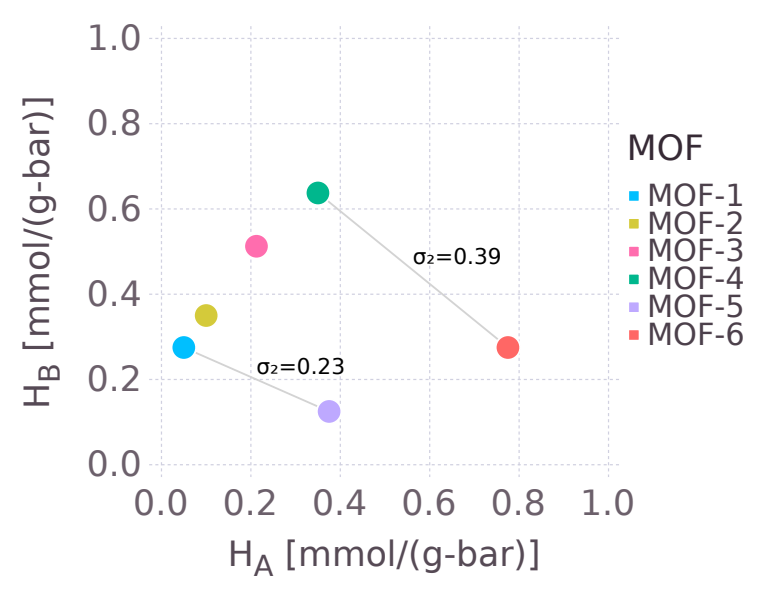

(b)

Figure S12: Choosing MOFs for a two-sensor array based on their adsorptive selectivities does not yield the optimal pair of MOFs for a robustly accurate sensor array. In both scatter plots (a) and (b), each point depicts the (hypothetical) Henry coefficients of gas species $A$ and $B, H_{A}$ and $H_{B}$ respectively, in a set of (a) three and (b) six (hypothetical) MOFs serving as candidates for a two-sensor array to determine the composition of an $A / B$ mixture. A gray line connecting a pair of MOFs is associated with the smallest singular value $\sigma_{2}(\mathbf{H})$ of the Henry coefficient matrix formed for that MOF pair. (a) Despite that MOF-1 offers the highest $B / A$ selectivity and MOF-2 offers the highest $A / B$ selectivity, the MOF pair yielding the largest $\sigma_{2}(\mathbf{H})$ is comprised of MOF-2 and MOF-3. (b) Despite that MOF-1 offers the highest $B / A$ selectivity and MOF-5 offers the highest $A / B$ selectivity, the MOF pair yielding the largest $\sigma_{2}(\mathbf{H})$ is comprised of MOF-4 and MOF-6. 


\section{S3 Color coding to visualize the linear maps $\mathrm{p}^{*} \mapsto \mathrm{m}^{*}$}

The idea to visualize the linear maps with color as in Fig. 4 emanated from domain coloring ${ }^{10}$ to visualize a complex function $f: \mathbb{C} \mapsto \mathbb{C}$. In domain coloring, each point in the complex plane $\mathbb{C}$ (the domain) is assigned a color and brightness (as a pair, unique to each point in $\mathbb{C}$ ). Typically, the phase determines the color, and a cyclic colormap is used to avoid a perceptual discontinuity in the color at some arbitrary phase. To visualize how a complex function $f$ maps a point $z \in \mathbb{C}$ into its image, another complex plane is drawn to represent the codomain; on this plot, each point $f(z)$ in the range of $f$ is then assigned the same color and brightness as the corresponding $z$ in the domain. We took inspiration from domain coloring and similarly (a) assigned a unique color to each angle on a circle in composition space and (b) colored a given $\mathbf{m}^{*}$ in response space - the image of some (perhaps multiple) $\mathbf{p}^{*}$ - as the same color as the corresponding $\mathbf{p}^{*}$ to visualize the mapping.

Computationally, to generate Fig. 2, we first label each $\mathbf{p}^{*}$ on the circle in composition space with a unique color from a cyclic colormap. Then, for each point $\mathbf{p}^{*}$ on this circle, when we compute the corresponding sensor array response $\mathbf{m}^{*}$ from $\mathbf{m}^{*}=\mathbf{H} \mathbf{p}^{*}$ and plot it in the response space, we assign $\mathbf{m}^{*}$ the same color as the $\mathbf{p}^{*}$ that mapped to there by $\mathbf{H}$. In the case of Fig. 2(a), two points on the circle in composition space were mapped to a single point in sensor response space (i.e., the mapping was not one-to-one). To handle this, we ensured that the two points in composition space that mapped to the same point in sensor response space were assigned the same color. 


\section{S4 Overdetermined systems: least squares and the normal equation}

In the case where we have an overdetermined system $\mathbf{m}=\mathbf{H p}$ (i.e., more (distinct) QCMMOF sensors than number of gas phase components $n>g$ ), we can use least squares to arrive at a prediction of the partial pressures of the gases. The least squares procedure is to choose the $\mathbf{p}$ such that the error, $\mathbf{e}(\mathbf{p})=\mathbf{m}-\mathbf{H p}$, is as close to the zero vector as possible:

$$
\mathbf{p}=\underset{\tilde{\mathbf{p}}}{\arg \min }\|\mathbf{m}-\mathbf{H} \tilde{\mathbf{p}}\|^{2} .
$$

The $\mathbf{p}$ that minimizes the error satisfies:

$$
\nabla_{\mathbf{p}}\|\mathbf{m}-\mathbf{H p}\|^{2}=\mathbf{0} .
$$

Note that:

$$
\begin{aligned}
\|\mathbf{m}-\mathbf{H} \mathbf{p}\|^{2} & =(\mathbf{m}-\mathbf{H} \mathbf{p})^{\top}(\mathbf{m}-\mathbf{H} \mathbf{p}) \\
& =\mathbf{m}^{\top} \mathbf{m}-2 \mathbf{p}^{\top} \mathbf{H}^{\top} \mathbf{m}+\mathbf{p}^{\top} \mathbf{H}^{\top} \mathbf{H} \mathbf{p}
\end{aligned}
$$

Since $\nabla_{\mathbf{p}}\left(\mathbf{m}^{\top} \mathbf{m}\right)=\mathbf{0}$, the minimizer $\mathbf{p}$ satisfies:

$$
\nabla_{\mathbf{p}}\left(-2 \mathbf{p}^{\top} \mathbf{H}^{\top} \mathbf{m}+\mathbf{p}^{\top} \mathbf{H}^{\top} \mathbf{H} \mathbf{p}\right)=\mathbf{0} .
$$

Recalling some matrix calculus, for a vector $\mathbf{x}$ and a matrix $\mathbf{A}$ :

$$
\nabla_{\mathbf{x}}\left(\mathbf{x}^{\top} \mathbf{A}\right)=\mathbf{A}
$$

and

$$
\nabla_{\mathbf{x}}\left(\mathbf{x}^{\top} \mathbf{A} \mathbf{x}\right)=\left(\mathbf{A}+\mathbf{A}^{\top}\right) \mathbf{x} .
$$


Applying matrix calculus to eqn. S5:

$$
-2 \mathbf{H}^{\top} \mathbf{m}+2 \mathbf{H}^{\top} \mathbf{H} \mathbf{p}^{*}=0 .
$$

This leads to the so-called normal equation which the minimizer $\mathbf{p}$ in eqn. S1 satisfies:

$$
\mathbf{H}^{\top} \mathbf{H p}=\mathbf{H}^{\top} \mathbf{m}
$$

\section{S5 On the singular values when a row is added to $\mathbf{H}$}

The singular values of $\mathbf{H}$ in eqn. 19 are the square roots of the eigenvalues of the $g \times g$ matrix $\mathbf{H}^{\top} \mathbf{H}$ :

$$
\mathbf{H}^{\top} \mathbf{H}=\left[\begin{array}{ll}
\mathbf{H}_{0}^{\top} & \mathbf{H}_{a}^{\top}
\end{array}\right]\left[\begin{array}{l}
\mathbf{H}_{0} \\
\mathbf{H}_{a}
\end{array}\right]=\mathbf{H}_{0}^{\top} \mathbf{H}_{0}+\mathbf{H}_{a}^{\top} \mathbf{H}_{a} .
$$

The matrix $\mathbf{H}_{0}^{\top} \mathbf{H}_{0}$ is symmetric, and $\mathbf{H}_{a}^{\top} \mathbf{H}_{a}$ is a positive semi-definite $g \times g$ matrix of rank one. Ref. ${ }^{11}$ claims that, because $\mathbf{H}^{\top} \mathbf{H}$ is a rank-one, positive semi-definite perturbation of the symmetric matrix $\mathbf{H}_{0}^{\top} \mathbf{H}_{0}$, the following relationship holds for the eigenvalues $\lambda_{i}$, where $\lambda_{1} \geq \lambda_{2} \geq \cdots \geq \lambda_{g}$

$$
\lambda_{i}\left(\mathbf{H}^{\top} \mathbf{H}\right) \geq \lambda_{i}\left(\mathbf{H}_{0}^{\top} \mathbf{H}_{0}\right) .
$$

for $i \in\{1,2, \ldots, g\}$. Since the singular values of a tall matrix $\mathbf{A}$ are the square roots of the (non-negative) eigenvalues of $\mathbf{A}^{T} \mathbf{A}$, we have shown:

$$
\sigma_{i}(\mathbf{H}) \geq \sigma_{i}\left(\mathbf{H}_{0}\right)
$$

for $i \in\{1,2, \ldots, g\}$. i.e., adding another sensor to a sensor array increases (or keeps constant) all singular values of the Henry coefficient matrix of the array. The implication is that $\left\langle\|\delta \mathbf{p}\|^{2}\right\rangle$ in eqn. 26 decreases or stays the same (a desirable situation) upon adding another sensor to the array. 


\section{References}

(1) Tchalala, M.; Bhatt, P.; Chappanda, K.; Tavares, S.; Adil, K.; Belmabkhout, Y.; Shkurenko, A.; Cadiau, A.; Heymans, N.; De Weireld, G.; Maurin, G.; Salama, K.; Eddaoudi, M. Fluorinated MOF platform for selective removal and sensing of $\mathrm{SO}_{2}$ from flue gas and air. Nature Communications 2019, 10, 1328.

(2) Carter, J. H.; Han, X.; Moreau, F. Y.; da Silva, I.; Nevin, A.; Godfrey, H. G.; Tang, C. C.; Yang, S.; Schroder, M. Exceptional Adsorption and Binding of Sulfur Dioxide in a Robust Zirconium-Based Metal-Organic Framework. Journal of the American Chemical Society 2018, 140, 15564-15567.

(3) Tan, K.; Canepa, P.; Gong, Q.; Liu, J.; Johnson, D. H.; Dyevoich, A.; Thallapally, P. K.; Thonhauser, T.; Li, J.; Chabal, Y. J. Mechanism of preferential adsorption of $\mathrm{SO}_{2}$ into two microporous paddle wheel frameworks $\mathrm{M}(\mathrm{bdc})(\mathrm{ted})_{0.5}$. Chemistry of Materials 2013, 25, 4653-4662.

(4) Mason, J. A.; Sumida, K.; Herm, Z. R.; Krishna, R.; Long, J. R. Evaluating metalorganic frameworks for post-combustion carbon dioxide capture via temperature swing adsorption. Energy \& Environmental Science 2011, 4, 3030-3040.

(5) Liang, Z.; Marshall, M.; Chaffee, A. L. $\mathrm{CO}_{2}$ adsorption, selectivity and water tolerance of pillared-layer metal organic frameworks. Microporous and Mesoporous Materials 2010, 132, 305-310.

(6) Savage, M. et al. Selective adsorption of sulfur dioxide in a robust metal-organic framework material. Advanced Materials 2016, 28, 8705-8711.

(7) Yang, S.; Liu, L.; Sun, J.; Thomas, K. M.; Davies, A. J.; George, M. W.; Blake, A. J.; Hill, A. H.; Fitch, A. N.; Tang, C. C.; Schrder, M. Irreversible network transformation in a dynamic porous host catalyzed by sulfur dioxide. Journal of the American Chemical Society 2013, 135, 4954-4957. 
(8) Yang, S.; Sun, J.; Ramirez-Cuesta, A. J.; Callear, S. K.; David, W. I.; Anderson, D. P.; Newby, R.; Blake, A. J.; Parker, J. E.; Tang, C. C.; Schrder, M. Selectivity and direct visualization of carbon dioxide and sulfur dioxide in a decorated porous host. Nature Chemistry 2012, 4, 887.

(9) Thallapally, P. K.; Motkuri, R. K.; Fernandez, C. A.; McGrail, B. P.; Behrooz, G. S. Prussian blue analogues for $\mathrm{CO}_{2}$ and $\mathrm{SO}_{2}$ capture and separation applications. Inorganic Chemistry 2010, 49, 4909-4915.

(10) Wegert, E.; Semmler, G. Phase plots of complex functions: a journey in illustration. Notices AMS 2010, 58, 768-780.

(11) Thompson, R. The behavior of eigenvalues and singular values under perturbations of restricted rank. Linear Algebra and its Applications 1976, 13, 69-78. 
